\title{
Atmospheric and Surface Contributions to Planetary Albedo
}

\author{
AARon Donohoe AND DAVID S. BATTISTI \\ Department of Atmospheric Sciences, University of Washington, Seattle, Washington
}

(Manuscript received 28 July 2010, in final form 7 March 2011)

\begin{abstract}
The planetary albedo is partitioned into a component due to atmospheric reflection and a component due to surface reflection by using shortwave fluxes at the surface and top of the atmosphere in conjunction with a simple radiation model. The vast majority of the observed global average planetary albedo (88\%) is due to atmospheric reflection. Surface reflection makes a relatively small contribution to planetary albedo because the atmosphere attenuates the surface contribution to planetary albedo by a factor of approximately 3 . The global average planetary albedo in the ensemble average of phase 3 of the Coupled Model Intercomparison Project (CMIP3) preindustrial simulations is also primarily (87\%) due to atmospheric albedo. The intermodel spread in planetary albedo is relatively large and is found to be predominantly a consequence of intermodel differences in atmospheric albedo, with surface processes playing a much smaller role despite significant intermodel differences in surface albedo. The CMIP3 models show a decrease in planetary albedo under a doubling of carbon dioxide-also primarily due to changes in atmospheric reflection (which explains more than $90 \%$ of the intermodel spread). All models show a decrease in planetary albedo due to the lowered surface albedo associated with a contraction of the cryosphere in a warmer world, but this effect is small compared to the spread in planetary albedo due to model differences in the change in clouds.
\end{abstract}

\section{Introduction}

The ratio of reflected to incident shortwave radiation at the top of the atmosphere (TOA), the earth's planetary albedo, is a function of climate state and exerts a profound influence on the earth's climate. As a reference point, Budyko (1969) postulated that a change in global average planetary albedo of less than 0.02 units could cause global glaciation of the climate system. The radiative forcing associated with a doubling of carbon dioxide above the preindustrial atmospheric concentration (3.7 $\mathrm{W} \mathrm{m}^{-2}$; Solomon et al. 2007) is approximately equivalent to a 0.01 -unit change in global average planetary albedo. The magnitude of temperature variability seen over the Holocene (Moberg et al. 2005) is consistent with planetary albedo variations of 0.01 units or less (Cahalan et al. 1994; Bender 2011).

The meridional structure of planetary albedo is also inextricably linked to the strength of the atmospheric and oceanic circulation. The large-scale circulation is primarily driven by the equator-to-pole gradient in net

Corresponding author address: Aaron Donohoe, University of Washington, 408 ATG Building, Box 351640, Seattle, WA 98195. E-mail: aaron@atmos.washington.edu shortwave radiation (Hartmann 1994), which owes approximately one-third of its existence to meridional gradient in planetary albedo (Stone 1978). Climate states with altered meridional structures of planetary albedo exhibit varying magnitudes of meridional heat transport (Enderton and Marshall 2009).

Finally, changes in planetary albedo associated with both cloud and surface processes are among the most powerful feedback processes in the response of the climate system to anthropogenic forcing (Bony et al. 2006), and variations in the planetary albedo feedback explain a large portion of the intermodel spread in climate sensitivity among global climate models (Bender et al. 2006). Understanding the processes that control the earth's planetary albedo in the current climate state is central to predicting how the planetary albedo will respond to future climate changes.

The planetary albedo is a function of the optical properties of objects within the atmosphere (e.g., clouds, water vapor, and aerosols) and objects that constitute the planet's surface (e.g., ice, ocean, and trees) (Wielicki et al. 1995; Hall 2004). It is convenient to lump the atmosphere's contribution to planetary albedo into three bulk processes: 1) clouds and aerosols that directly reflect incident solar radiation back to space, 
2) atmospheric opacity to downwelling shortwave radiation that limits the amount of downwelling shortwave radiation reaching the surface, and 3) atmospheric opacity to shortwave radiation upwelling from the surface that limits the amount of shortwave radiation reflected by the surface that escapes to space (Qu and Hall 2005). Thus, the atmosphere influences the planetary albedo by way of direct reflection back to space (process 1) and by attenuating the effect of surface albedo on planetary albedo (processes 2 and 3). Both the atmospheric and surface contributions to planetary albedo are functions of the climate state.

Several studies have examined the surface contribution to local planetary albedo in the polar regions. Gorodetskaya et al. (2006) used the satellite record of planetary albedo in conjunction with observational datasets of snow and ice concentration to demonstrate that changing ice/snow concentrations from $0 \%$ to $100 \%$ would result in local planetary albedo changes of order 0.20 with some regional dependence. This number is significantly less than the change in surface albedo associated with the melting of the cryosphere (Hummel and Reck 1979), which suggests that the atmosphere strongly attenuates the impact of the surface albedo change on the planetary albedo. Qu and Hall (2005) found that surface reflection accounts for less than $25 \%$ of the climatological planetary albedo in the ice- and snow-covered regions of the planet and the remainder is due to clouds. They also found that, although the year-toyear variability of planetary albedo in cryospheric regions is mainly due to changes in surface albedo, atmospheric processes attenuate the effect of the surface albedo changes on the local planetary albedo by as much as $90 \%$ (i.e., the change in planetary albedo is $10 \%$ of the change in surface albedo).

In this study, we quantify the relative contributions of the surface and atmosphere to planetary albedo in the observations (section 2). We then perform the same analyses on the output of the preindustrial integrations from the climate models used in the International Panel on Climate Change (IPCC) Fourth Assessment Report (AR4) to determine whether the relative contributions of surface and atmospheric processes to the total planetary albedo observed in nature is captured in the model simulations. We also calculate the intermodel spread in the hemispheric average planetary albedo and determine what portion of this spread is due to surface or atmospheric processes (section 3). Finally, we analyze the changes in planetary albedo in the AR4 climate models due to a doubling of atmospheric carbon dioxide concentration and partition the changes in planetary albedo between changes in atmospheric and surface processes. We also examine planetary albedo in vastly different climate states, ranging from the last glacial maximum to an ice-free aquaplanet (section 4). A summary and discussion of our results follows.

\section{Partitioning of planetary albedo in nature}

In this section, we partition the observed planetary albedo into atmospheric and surface components and assess the contribution of each component to the hemispheric average planetary albedo.

\section{a. Datasets used}

We use the TOA and surface shortwave radiation data products from the Clouds and Earth's Radiant Energy System (CERES) experiment (Wielicki et al. 1996). We use Fasullo and Trenberth's (2008a,b) long-term climatologies of the CERES TOA data that correct for missing data and global average energy imbalances. For the surface shortwave fluxes, we use the CERES "AVG" fields, which are derived by assimilating the satellite observations into a radiative transfer model to infer the surface fluxes (Rutan et al. 2001). All calculations are performed separately for each of the four CERES instruments (FM1 and FM2 on Terra from 2000 to 2005 and FM3 and FM4 on Aqua from 2002 to 2005). We then average the results over the four instruments. Our calculations are performed on the annual average (solar weighted) data.

The observed TOA fluxes from CERES differ from previous satellite measurements of the Earth Radiation Budget Experiment (ERBE; Barkstrom et al. 1989) by as much as $8 \mathrm{~W} \mathrm{~m}^{-2}$ in the zonal average (Trenberth et al. 2009; Loeb et al. 2009). The instrumental absolute calibration error in the CERES TOA fluxes are as large as $4.2 \mathrm{~W} \mathrm{~m}^{-2}$ (Loeb et al. 2009) and the total error in the global average shortwave flux is estimated to be $2.6 \mathrm{~W} \mathrm{~m}^{-2}$ (Bender et al. 2006). Similarly, the CERES surface shortwave fluxes have systematic errors ranging from $3 \%$ to $5 \%$ (Kratz et al. 2010) and random errors in the hourly retrievals of order tens of watts per square meter (see Trenberth et al. 2009 for a discussion). In appendix A, we propagate the errors in the observational fluxes to obtain error estimates for the quantities calculated in this study.

\section{b. Partitioning planetary albedo into atmospheric and surface components}

\section{1) Methodology}

We now describe a methodology for partitioning the planetary albedo into atmospheric and surface components, given the upwelling and downwelling solar fluxes at both the TOA and the surface. We will denote the 
atmospheric contribution to the planetary albedo as $\alpha_{P, \text { ATMOS }}$ and the surface contribution to planetary albedo as $\alpha_{P, \text { SURF }}$ in the remainder of this paper.

At each grid point we build a single-layer model of solar radiation that accounts for three shortwave processes: atmospheric reflection, atmospheric absorption, and surface reflection. We assume that each of these processes is isotropic: a certain percentage of the incident radiation is absorbed per pass through the atmosphere and a different percentage of the incident radiation is reflected per pass through the atmosphere. For example, of the total downwelling solar radiation incident at the TOA $S$, a fraction $R$ is reflected by the atmosphere, a fraction $A$ is absorbed by the atmosphere, and the remainder is transmitted to the surface. Of the transmitted radiation, a fraction $\alpha$ (the surface albedo) is reflected at the surface back toward the atmosphere. Of this reflected radiation, a portion $R$ is reflected back to the surface by the atmosphere, a portion $A$ is absorbed within the atmosphere, and the remainder is transmitted to space (Fig. 1). These processes are repeated for an infinite number of reflections. Hence, the annual mean upwelling solar flux at each grid point at the TOA is

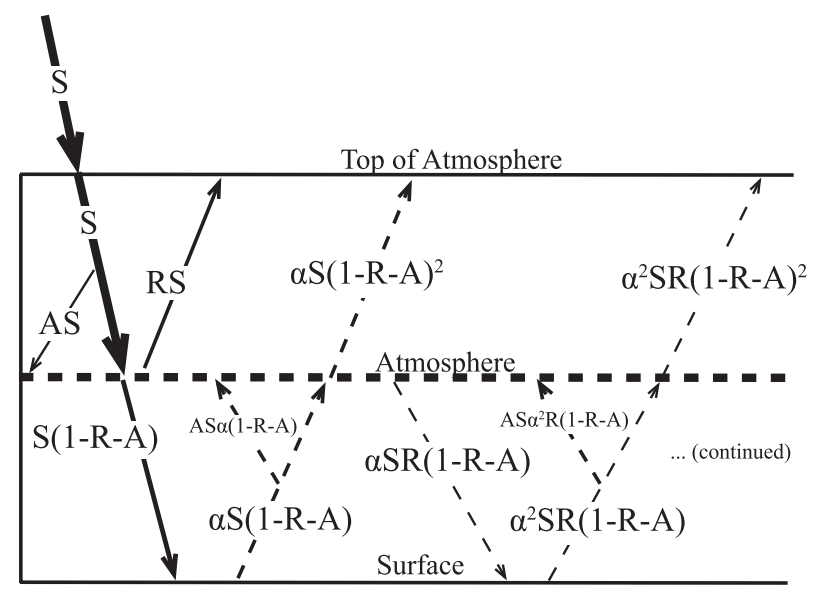

FIG. 1. Schematic representing the first two reflections in the single-layer solar radiation model. Moving from left to right, the arrows represent the radiative fluxes associated with the incident solar, first reflection, and second reflection. The variables $A, R$, and $\alpha$ are the atmospheric absorption fraction during a single pass through the atmosphere, the fraction of cloud reflection, and the surface albedo, respectively. The solid arrows at the TOA represent the radiative fluxes that we associated with cloud reflection and the dashed lines represent the radiative fluxes that we associated with the surface reflection.

$$
\begin{aligned}
F \uparrow_{\mathrm{TOA}} & =S\left[R+\alpha(1-R-A)^{2}+\alpha^{2} R(1-R-A)^{2}+\alpha^{3} R^{2}(1-R-A)^{2} \cdots\right] \\
& =S R+S \alpha(1-R-A)^{2}\left[1+(\alpha R)+(\alpha R)^{2} \cdots\right]=S R+S \alpha \frac{(1-R-A)^{2}}{1-\alpha R}
\end{aligned}
$$

where $F \uparrow_{\mathrm{TOA}}$ is the upwelling solar flux at the TOA and the convergence of the infinite series to the final expression on the right-hand side is ensured because both $R$ and $\alpha$ are less than one (Qu and Hall 2005). Similar convergent infinite series can be obtained for the downwelling and upwelling solar fluxes at the surface:

$$
\begin{aligned}
& F \downarrow_{\mathrm{SURF}}=S \frac{(1-R-A)}{1-\alpha R}, \quad \text { and } \\
& F \uparrow_{\mathrm{SURF}}=\alpha S \frac{(1-R-A)}{1-\alpha R}=\alpha F \downarrow_{\mathrm{SURF}} .
\end{aligned}
$$

Therefore, given datasets of shortwave radiative fluxes on the left-hand side of Eqs. (1)-(3) and $S$, these equations represent a system of three equations in terms of three unknown variables: $A, R$, and $\alpha$ (see Table 1 for descriptions of all variables). In practice, the ratio of upwelling to downwelling radiation at the surface [Eqs. (3) and (2)] defines $\alpha$ such that the system can be reduced to two equations [Eqs. (1) and (2)] and two unknowns $(A$ and $R$ ). One can show that all possible solutions to our equations have $0 \leq R \leq 1$ and $0 \leq A \leq 1$, although it is not clear to us whether a solution to the generalized system of equations must exist. Nonetheless, solutions to Eqs. (1)-(3) exist at all grid points for the datasets and GCM output discussed in this paper. Furthermore, all solutions ( $A$ and $R$ values at each grid point) discussed here are unique.

Solving these equations results in spatial maps of $R$ (Fig. 2d) and $A$ (not shown). Dividing Eq. (1) by $S$ and separating the two terms allows us to partition the planetary albedo into $\alpha_{P, \mathrm{ATMOS}}$ and $\alpha_{P, \mathrm{SURF}}$ components:

TABLE 1. Variables used in this study.

\begin{tabular}{ll}
\hline \hline Symbol & \multicolumn{1}{c}{ Meaning } \\
\hline$\alpha$ & Surface albedo \\
$\alpha_{P}$ & Planetary albedo = TOA albedo \\
$A$ & Percentage of absorption during each \\
& pass through the atmosphere \\
$R$ & Percentage of reflection during each \\
& pass through the atmosphere \\
$\alpha_{P, \text { ATMOS }}$ & Atmospheric contribution to planetary albedo \\
$\alpha_{P, \mathrm{SURF}}$ & Surface contribution to planetary albedo \\
$\chi$ & Atmospheric attenuation of surface albedo \\
\hline
\end{tabular}



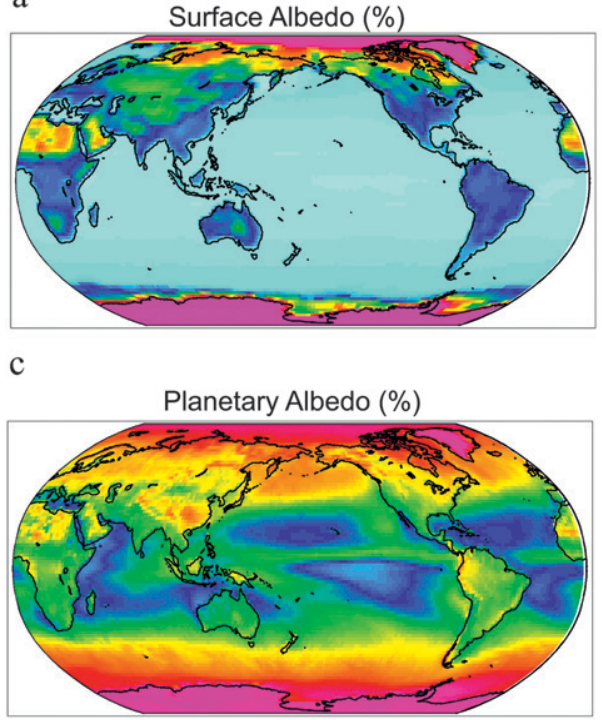

$\mathrm{b}$

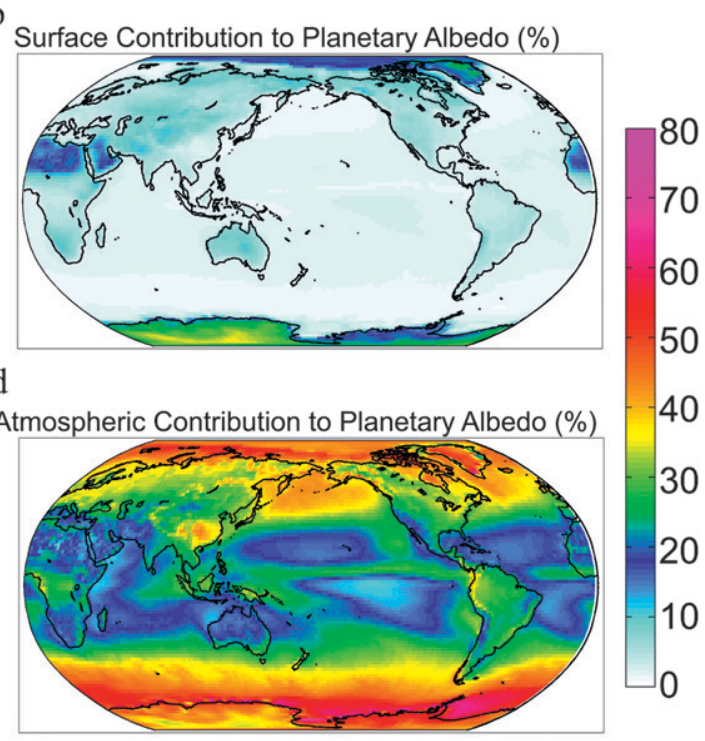

FIG. 2. (a) Surface and (c) planetary albedo, and (b) surface and (d) atmospheric contributions to (c). All quantities are expressed as a percentage, where $1 \%$ corresponds to 0.01 units of albedo.

$$
\begin{aligned}
\alpha_{P, \mathrm{ATMOS}} & =R, \text { and } \\
\alpha_{P, \mathrm{SURF}} & =\alpha \frac{(1-R-A)^{2}}{1-\alpha R} .
\end{aligned}
$$

Equation (4) is due to direct reflection by the atmosphere [the first term on the right-hand side of Eq. (1)]. All of the solar radiation that is reflected by the surface and eventually passes through the TOA [the second term on the right-hand side of Eq. (1)] is attributed to $\alpha_{P \text {,SURF. }}$ By definition, the surface and atmospheric contributions to planetary albedo sum to the planetary albedo:

$$
\alpha_{P}=\alpha_{P, \mathrm{ATMOS}}+\alpha_{P, \mathrm{SURF}} \cdot
$$

Maps of $\alpha_{P, \text { SURF }}$ and $\alpha_{P, \text { ATMOs }}$ are shown in Figs. $2 \mathrm{~b}, \mathrm{~d}$.

We calculate $\alpha_{P \text {,ATMOS }}$ and $\alpha_{P \text {,SURF }}$ using annual average (solar weighted) data. We have also performed the partitioning on the climatological monthly mean data and then averaged the monthly values of $\alpha_{P, \mathrm{ATMOS}}$ and $\alpha_{P, \mathrm{SURF}}$ to obtain the annual average climatology. The annual and zonal average $\alpha_{P, \text { ATMOS }}$ calculated from the monthly data agree with that calculated directly from the annual average data to within $1 \%$ of $\alpha_{P \text {,ATMOs }}$ at all latitudes.

We note that Taylor et al. (2007, hereafter T07) used a similar simplified radiative transfer model to partition planetary albedo into surface and atmospheric components. In contrast to our formulation, T07 assumed absorption only occurs on the first downward pass through the atmosphere and occurs above the level of atmospheric reflection. The impact of the differences in model formulation on the derived quantities $\alpha_{P, \text { ATMOS }}$ and $\alpha_{P, \mathrm{SURF}}$ are reported in appendix $\mathrm{B}$. The qualitative conclusions found in this study are independent of the assumptions made in the simplified radiative transfer model.

\section{2) RESUltS}

The maps of surface and planetary albedo exhibit large values in the polar regions, with larger spatial differences in the meridional direction than in the zonal direction (Fig. 2); the predominant spatial structure in both maps is an equator-to-pole gradient. Significant meridional gradients in surface albedo are constrained to be at the transition to the cryospheric regions (around $70^{\circ}$ in each hemisphere), whereas the meridional gradients in planetary albedo are spread more evenly across the storm-track regions (from $30^{\circ}$ to $60^{\circ}$ ).

The percentage of solar radiation absorbed during a single pass through the atmosphere (not shown) features a predominant equator-to-pole gradient with tropical values of order $25 \%$ and high-latitude values of order $15 \%$ with still smaller values occurring over the highest topography. The global pattern of atmospheric solar absorption is virtually identical to the pattern of vertically integrated specific humidity [from National Centers for Environmental Prediction (NCEP) reanalysis] with a spatial correlation coefficient of 0.92 ; this is expected because the atmospheric absorption of solar radiation is predominantly due to water vapor and ozone (Chou and Lee 1996) while clouds and black carbon each contribute less than $10 \%$ of the atmospheric absorption (Kim and Ramanathan 2008). 
The map of $\alpha_{P, \text { ATMOS }}$ (Fig. 2d) shows several anticipated features: 1) high values associated with the intertropical convergence zone (ITCZ) in the equatorial Pacific, 2) low values in the drier subtropics, 3) high values in the NH Atlantic and Pacific storm tracks and in the nearly zonally symmetric SH storm track associated with synoptic storms, 4) high values in the Arctic and West Antarctica that tend to be cloudy during the local summer (Curry et al. 1996), and 5) high values over Southeast Asia associated with extensive cold season stratocumulus (Hahn and Warren 2003).

As is true for the planetary albedo and $\alpha_{P, \mathrm{ATMOS}}$, $\alpha_{P, \mathrm{SURF}}$ is greater in the polar regions than in the tropics. More important, however, is that $\alpha_{P, \mathrm{SURF}}$ is greatly attenuated by the atmosphere (cf. Figs. $2 \mathrm{a}$ and $2 \mathrm{~b}$ ) - so much so that almost everywhere the planetary albedo is largely determined by the atmospheric and not the surface contribution (cf. Figs. $2 b$ and $2 d$ ). Our simple radiative model predicts that $\alpha_{P, \mathrm{SURF}}$ will be attenuated by square of the atmospheric transmissivity: $(1-R-A)^{2}$ [Eq. (5) and Fig. 1]. In this framework, we can see that while the atmospheric opacity primarily acts to damp the impact of the surface albedo on planetary albedo, the atmosphere also imparts some spatial structure to $\alpha_{P, \mathrm{SURF}}$ by virtue of the spatial structure in atmospheric opacity. For example, the surface albedo over Antarctica is fairly uniform, but $\alpha_{P, \mathrm{SURF}}$ is much larger over East Antarctica because the atmosphere over East Antarctica is less opaque (both less cloud and less absorption by water vapor) than that over West Antarctica.

Atmospheric processes play a much greater role than surface processes in determining the amplitude and structure of planetary albedo, both regionally and in the global average. The (solar weighted) global average planetary albedo of 0.298 is partitioned into a global average $\alpha_{P, \text { ATMOS }}$ of 0.262 ( $88 \%$ of the total) and a global average $\alpha_{P, \mathrm{SURF}}$ of 0.036 (12\%; Table 2). These results are in qualitative agreement with $\mathrm{Qu}$ and Hall's (2005) conclusion that "the atmosphere accounts for
TABLE 2. Global average planetary albedo, its decomposition into atmospheric and surface components (as described in the text), and global average surface albedo for the observations and the CMIP3 multimodel average and spread $(2 \sigma)$. The observational uncertainty on total planetary albedo is taken from Bender et al. (2006). The observational uncertainty on $\alpha_{P, \text { ATMOS }}$ and $\alpha_{P, \mathrm{SURF}}$ is assessed by the method described in appendix A. All entries are percentages.

\begin{tabular}{lrccc}
\hline \hline & $\alpha_{P}$ & $\alpha_{P, \text { ATMOS }}$ & $\alpha_{P, \text { SURF }}$ & $\alpha$ \\
\hline Obs & 29.8 & 26.2 & 3.6 & 12.3 \\
Observational uncertainty $(2 \sigma)$ & 0.8 & 1.8 & 0.9 & - \\
Model average & 30.4 & 26.4 & 4.0 & 13.2 \\
Model spread $(2 \sigma)$ & 1.6 & 2.3 & 1.3 & 3.1 \\
\hline
\end{tabular}

much more of the climatological planetary albedo $(\geq 75 \%)$ than the surface." The hemispheric average planetary albedo is very similar in both hemispheres ( 0.299 in the $\mathrm{NH}$ versus 0.298 in the $\mathrm{SH}$ ), although the partitioning between $\alpha_{P, \mathrm{ATMO}}$ and $\alpha_{P, \mathrm{SURF}}$ differs slightly between the hemispheres $\left(\alpha_{P, \mathrm{ATMO}}\right.$ contributes $86 \%$ of the hemispheric average planetary albedo in the $\mathrm{NH}$ as compared to $90 \%$ in the $\mathrm{SH}$ ).

The zonal average planetary albedo, partitioned into $\alpha_{P, \mathrm{ATMOS}}$ and $\alpha_{P, \mathrm{SURF}}$, is shown in Fig. 3a. At all latitudes, $\alpha_{P, \mathrm{ATMOS}}$ is greater than $\alpha_{P \text {,SURF }}$ (higher values of $\alpha_{P, \mathrm{SURF}}$ are over the cryospheric and NH landmass regions, owing to the high surface albedo in those regions). The largest values of $\alpha_{P \text {.ATMOS }}$ are in the stormtrack regions and the Arctic where clouds are prevalent, and the smallest values are in the dry subtropics; both the meridional structure of cloud fraction and solar zenith angle (Minnett 1999) contribute to the meridional structure of $\alpha_{P, \mathrm{ATMOS}}$.

As can be seen in Fig. 3b (or by comparing Figs. 2a and 2b), $\alpha_{P, \mathrm{SURF}}$ is much smaller than $\alpha$, because 1) the downwelling solar radiation at the TOA is attenuated by atmospheric reflection and absorption as it passes downward through the atmosphere and 2) the solar radiation reflected by the surface is attenuated by
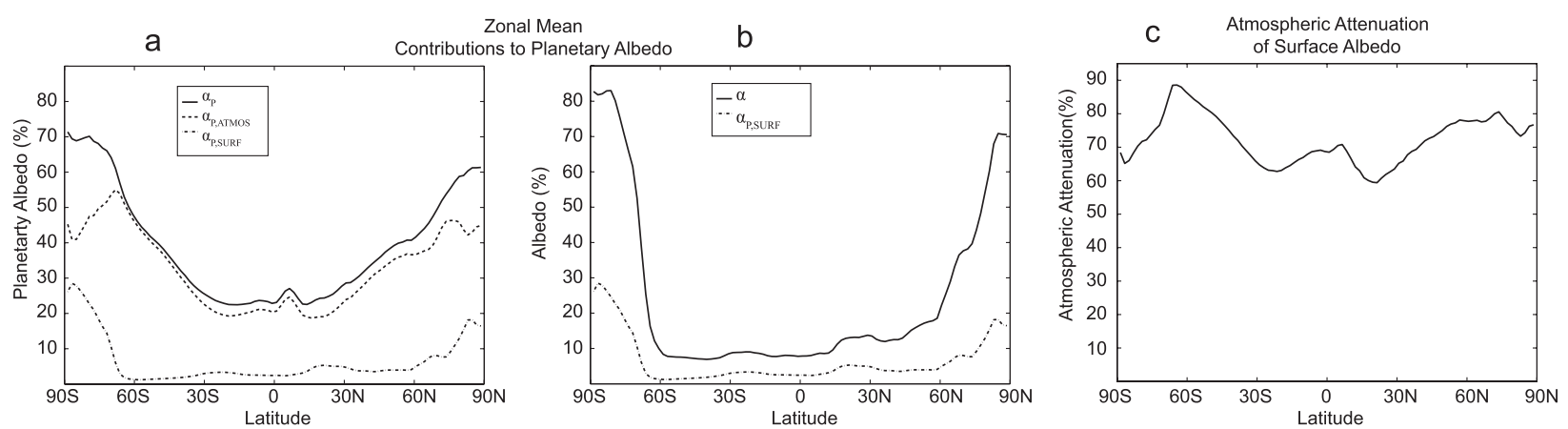

FIG. 3. (a) Zonal mean planetary albedo partitioned between atmospheric and surface components. (b) Zonal mean surface albedo and surface component of planetary albedo. (c) Atmospheric attenuation of surface albedo. 
atmospheric absorption and reflection as it passes upward from the surface to the TOA. Even over the polar regions, where the zonal mean surface albedo exceeds $70 \%, \alpha_{P, \text { SURF }}$ never exceeds $30 \%$. We define the atmospheric attenuation of surface albedo $\chi$ as

$$
\chi=1-\frac{\alpha_{P, \mathrm{SURF}}}{\alpha}=1-\frac{(1-R-A)^{2}}{1-R \alpha} .
$$

The atmospheric attenuation of the surface albedo (Fig. $3 \mathrm{c}$ ) ranges from a maximum of $80 \%-85 \%$ in the storm tracks to a minimum of $60 \%$ in the dry subtropics. The meridional variations in atmospheric attenuation of surface albedo are small compared to the global mean atmospheric attenuation despite the large meridional variations in cloud properties; this is partly because solar radiation is attenuated by clear-sky processes (i.e., solar atmospheric absorption), which contribute as much as $50 \%$ of the atmospheric attenuation in the global mean (Bony et al. 2006).

Over the observational period analyzed in this study, atmospheric processes are found to be the dominant $(88 \%)$ contributor to global average planetary albedo while surface processes make a much smaller contribution to the global average planetary albedo.

\section{Partitioning of planetary albedo in the models used in IPCC AR4}

In this section, we look at the intermodel spread in planetary albedo in the preindustrial (PI) simulations of the climate system performed using the climate models that were used in the IPCC AR4. We then employ the same methodology used in section 2 to partition planetary albedo into its atmospheric and surface components. We find, not surprisingly, that the intermodel spread in planetary albedo is due to the differences in atmospheric processes and only weakly related to differences in surface albedo.

\section{a. Model runs analyzed}

We use data from the World Climate Research Programme's (WCRP) Coupled Model Intercomparison Project phase 3 (CMIP3) multimodel dataset: an archive of a suite of standardized coupled simulations from 25 global climate models that were included in the International Panel on Climate Change's Fourth Assessment Report (https://esgcet.llnl.gov:8443/index.jsp). The set of model simulations is commonly referred to as the WCRP's CMIP3 multimodel dataset (Meehl et al. 2007). We use output from the PI simulations, which are forced with temporally invariant external forcing $\left(\mathrm{CO}_{2}\right.$ is set to 280 ppm). We use 15 coupled models that provided the output fields required for our analysis (Table 3 ).

\section{b. Results}

Averaged over all the CMIP3 models, the global average planetary albedo is 0.304 (Table 2). This value is within the range of observational estimates based on the corrected ERBE data (0.313) and CERES data (0.298) (see Fasullo and Trenberth 2008a,b and Loeb et al. 2009 for a thorough discussion of the different satellite datasets and their uncertainties). The intermodel spread (two standard deviations or $2 \sigma$ ) in planetary albedo is 0.016 , corresponding to a TOA net shortwave difference of approximately $5.5 \mathrm{~W} \mathrm{~m}^{-2}$ across the models. Averaged across the models, $\alpha_{P, \text { ATMOs }}$ contributes $87 \%$ of the global average planetary albedo, which agrees with the observed partitioning of global average planetary albedo between atmospheric and surface processes.

As is true of the observations, the two hemispheres contribute nearly equally to the global average planetary albedo in the models (spread on the ordinate of Fig. 4). Also in agreement with the observations, $\alpha_{P, \mathrm{SURF}}$ contributes slightly more to the hemispheric average planetary albedo in the $\mathrm{NH}$ than in the $\mathrm{SH}$ (the offset on abscissa of Fig. 4b between the $\mathrm{NH}$ and $\mathrm{SH}$ data).

The intermodel spread in hemispheric average $\alpha_{P, \text { ATMOS }}(2 \sigma=0.022)$ is approximately twice as large as that of $\alpha_{P, \mathrm{SURF}}(2 \sigma=0.012)$. Overall, the intermodel spread of hemispheric average planetary albedo is well correlated with the intermodel spread in hemispheric average $\alpha_{P \text {,ATMOS }}$ (Fig. 4a; $R^{2}=0.53$ and 0.71 in the NH and $\mathrm{SH}$ ); it is not correlated with the hemispheric average $\alpha_{P \text { SURF }}$ (Fig. 4b). The hemispheric average planetary albedo is poorly correlated with the hemispheric average $\alpha_{P \text {,SURF }}$ because the spread in hemispheric average $\alpha_{P, \mathrm{SURF}}$ is small compared to the spread in $\alpha_{P, \text { ATMOs. }}$ Furthermore, the intermodel spreads in $\alpha_{P, \mathrm{ATMOS}}$ and $\alpha_{P, \mathrm{SURF}}$ are negatively correlated as expected from Eq. (8) and discussed later in this section.

The large intermodel spread in global and hemispheric average planetary albedo is primarily due to atmospheric processes as opposed to surface albedo for three reasons: 1) the climatological planetary albedo is primarily $(87 \%)$ determined by atmospheric reflection, 2 ) the surface albedo contribution to planetary albedo is strongly attenuated by the atmospheric opacity, and 3) a portion of the intermodel spread in surface albedo is a consequence of the intermodel spread in atmospheric opacity. As a consequence, the hemispheric average surface albedo is very weakly correlated with hemispheric average planetary albedo $\left(R^{2}=0.03\right.$ in the $\mathrm{NH}$ and 0.12 in the SH; Fig. 5) despite the relatively large intermodel spread in hemispheric average surface albedo $(2 \sigma=0.022$ in the $\mathrm{NH}$ and 0.036 in the $\mathrm{SH})$. 
TABLE 3. Models used in this study and their resolution. The horizontal resolution refers to the latitudinal and longitudinal grid spacing or the spectral truncation. The vertical resolution is the number of vertical levels.

\begin{tabular}{|c|c|c|c|}
\hline Abbreviation & Full name (Country) & $\begin{array}{l}\text { Horizontal } \\
\text { resolution }\end{array}$ & $\begin{array}{l}\text { Vertical } \\
\text { resolution }\end{array}$ \\
\hline BCCR-BCM2.0 & $\begin{array}{l}\text { Bjerknes Centre for Climate Research Bergen Climate Model } \\
\text { version } 2.0 \text { (Norway) }\end{array}$ & T63 & L31 \\
\hline CCCma-CGCM3.1 & $\begin{array}{l}\text { Canadian Centre for Climate Modelling and Analysis Coupled } \\
\text { General Circulation Model, version 3.1 (Canada) }\end{array}$ & $\mathrm{T} 47$ & L31 \\
\hline CNRM-CM3 & $\begin{array}{l}\text { Centre National de Recherches Météorologiques Coupled Global } \\
\text { Climate Model, version } 3 \text { (France) }\end{array}$ & T63 & L45 \\
\hline CSIRO-MK3.0 & $\begin{array}{l}\text { Commonwealth Scientific and Industrial Research Organisation Mark } \\
\text { version 3.0 (Australia) }\end{array}$ & T63 & L18 \\
\hline GFDL-CM2.0 & $\begin{array}{l}\text { National Oceanic and Atmospheric Administration (NOAA)/Geophysical } \\
\text { Fluid Dynamics Laboratory GFDL Climate Model version 2.0 (USA) }\end{array}$ & $2.0^{\circ} \times 2.5^{\circ}$ & $\mathrm{L} 24$ \\
\hline GISS-ER & $\begin{array}{l}\text { National Aeronautics and Space Administration (NASA)/Goddard } \\
\text { Institute for Space Studies Model E-R (USA) }\end{array}$ & $4^{\circ} \times 5^{\circ}$ & L20 \\
\hline IAP-FGOALS & $\begin{array}{l}\text { Institute of Atmospheric Physics Flexible Global Ocean- } \\
\text { Atmosphere-Land System Model, } \\
\text { National Key Laboratory of Numerical Modeling for Atmospheric } \\
\text { Sciences and Geophysical Fluid Dynamics (LASG) (China) }\end{array}$ & $\mathrm{T} 42$ & L26 \\
\hline MPI-ECHAM5 & Max Planck Institute for Meteorology/ECHAM5 (Germany) & T63 & L31 \\
\hline INM-CM3.0 & Institute of Numerical Mathematics Coupled Model, version 3.0 (Russia) & $4^{\circ} \times 5^{\circ}$ & $\mathrm{L} 21$ \\
\hline IPSL CM4.0 & L'Institut Pierre-Simon Laplace Coupled Model, version 4 (France) & $2.5^{\circ} \times 3.75^{\circ}$ & L19 \\
\hline Miroc3.2(hires) & $\begin{array}{l}\text { Model for Interdisciplinary Research on Climate 3.2, high-resolution } \\
\text { version (Japan) }\end{array}$ & T106 & L56 \\
\hline MRI-CGCM2.3.2a & $\begin{array}{l}\text { Meteorological Research Institute Coupled General Circulation Model, } \\
\text { version 2.3.2a (Japan) }\end{array}$ & $\mathrm{T} 42$ & L30 \\
\hline NCAR-CCSM3.0 & NCAR Community Climate System Model, version 3 (USA) & T85 & L26 \\
\hline UKMO HadCM3 & Third climate configuration of the Met Office Unified Model (UK) & $2.5^{\circ} \times 3.8^{\circ}$ & L19 \\
\hline MIUB-ECHOg & $\begin{array}{l}\text { Meteorological Institute of the University of Bonn, ECHO-G } \\
\text { Model (Germany) }\end{array}$ & $\mathrm{T} 30$ & L19 \\
\hline
\end{tabular}

The spatial map of the intermodel average planetary albedo is very similar to that observed except in Saharan Africa, the Arabian Peninsula, and the subtropical stratocumulus regions (not shown); in these regions, the models are biased low (the difference exceeds $2 \sigma$ ) compared to the observations. This bias exceeds the observational uncertainty in planetary albedo over those regions (Loeb et al. 2009). The intermodel spread in planetary albedo is greatest in the midlatitude stormtrack regions over the North Atlantic, North Pacific, and Southern Ocean and in the cryosphere regions of both hemispheres (not shown).
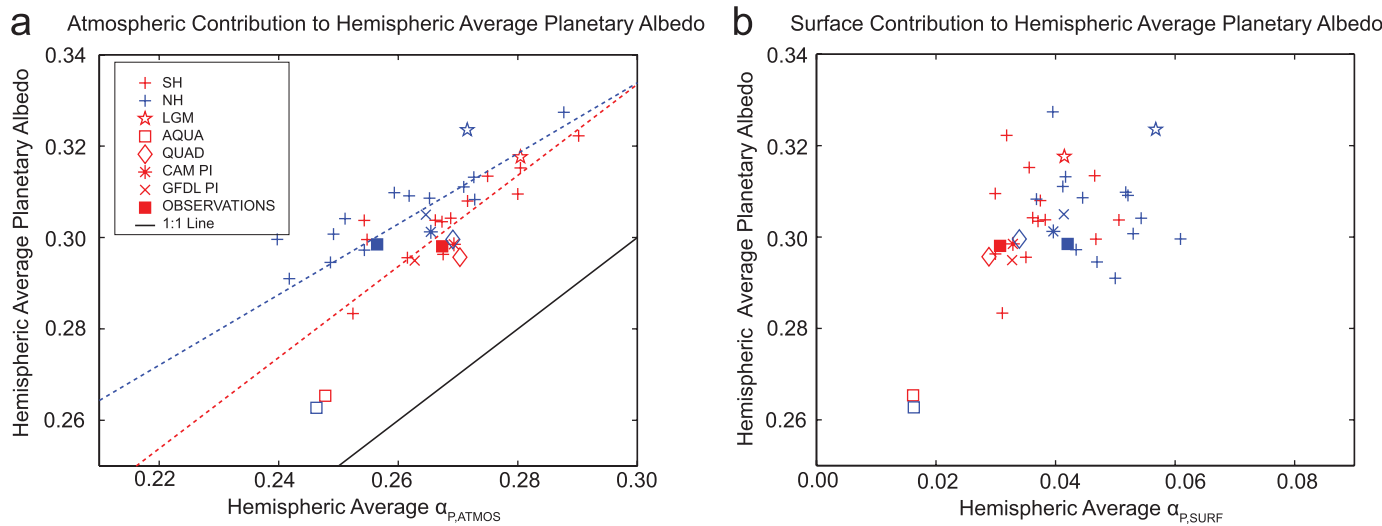

FIG. 4. (a) Hemispheric average $\alpha_{P}$ vs hemispheric average $\alpha_{P, \text { ATMOS }}$ in the CMIP3 PI simulations (crosses), observations (solid squares), and altered climate states. Red symbols and lines are for the SH while blue denotes the NH. The dashed lines are linear best fits to the PI simulations and the solid line is the 1:1 line with zero intercept. (b) As in (a), except plotted against hemispheric average $\alpha_{P, \mathrm{SURF}}$. 


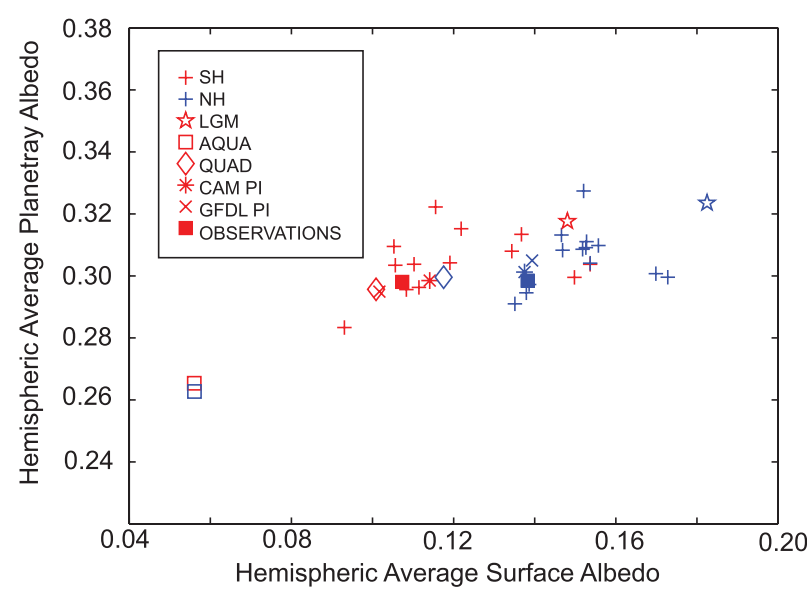

FIG. 5. Hemispheric average $\alpha_{P}$ vs hemispheric average $\alpha$ in the PI CMIP3 simulation (crosses), observations (solid squares), and altered climate states. Red symbols and lines are for the SH while blue denotes the NH. The axes have a 1:1 ratio.

The zonal and annual average surface albedo, planetary albedo, $\alpha_{P, \mathrm{ATMOS}}$, and $\alpha_{P, \mathrm{SURF}}$ for each member of the CMIP3 PI ensemble are coplotted (dashed black lines) in Figs. 6a-d alongside the observations (light blue lines). (Results from some additional model experiments are also shown in Fig. 6 and will be discussed in section 4.) In agreement with the observations, the $\alpha_{P, \mathrm{ATMO}}$ in the CMIP3 ensemble is larger than $\alpha_{P, \mathrm{SURF}}$ at all latitudes with the exception of the high Arctic and Antarctic (cf. Figs. 6c and 6d). The meridional structure of surface albedo is consistent with the observations. However, there are substantial differences between models in annual average surface albedo, especially in the cryosphere regions as discussed in Roesch (2006).

The zonal average planetary albedo (Fig. 6b) exhibits substantial intermodel spread, exceeding 0.08 units $(2 \sigma)$ in the high latitudes (Fig. 7), which is attributed to differences in $\alpha_{P, \mathrm{ATMOS}}$ in our analysis. Only in the high polar regions does $\alpha_{P, \mathrm{SURF}}$ contribute significantly to the intermodel spread in planetary albedo (Fig. 7). This result suggests that model differences in the meridional structure of planetary albedo are primarily determined by atmospheric (cloud) processes and only weakly by intermodel differences in surface processes.

With the exception of the SH ice margin, the intermodel standard deviation of $\alpha_{P, \text { ATMOS }}$ exceeds the total intermodel standard deviation of planetary albedo (Fig. 7); this result is a consequence of the negative correlation between $\alpha_{P, \mathrm{ATMOS}}$ and $\alpha_{P, \mathrm{SURF}}$ (not shown) that was also noted above. The negative correlation is expected based on our solar radiation model [i.e., Eq. (5)]; if two models had identical surface albedo in the Arctic but different cloud reflectance (and hence atmospheric transmissivity) the model with the larger atmospheric reflectance would have a reduced $\alpha_{P \text {,SURF }}$ because the reflective surface is less visible from the TOA. This result is interesting because it suggests that a portion of the intermodel spread in $\alpha_{P, \mathrm{SURF}}$ is due to atmospheric variability across models as opposed to the intermodel spread of surface albedo.

\section{Planetary albedo in simulations of altered climate states}

In this section, we analyze the change in planetary albedo and its partitioning between surface and atmospheric processes due to a doubling of carbon dioxide from the preindustrial values and in climate states with severely altered surface albedo distributions.

\section{a. Carbon dioxide doubling experiments}

\section{1) MOdel RunS USED}

Here we analyze the output from the "1pctto2x" simulations in the CMIP3 archive (Meehl et al. 2007). The initial conditions for each model come from the equilibrated PI simulations. Atmospheric $\mathrm{CO}_{2}$ is increased at $1 \% \mathrm{yr}^{-1}$ until $\mathrm{CO}_{2}$ has doubled relative to the PI concentration $(70 \mathrm{yr})$. The simulations are then run forward for an additional $150 \mathrm{yr}$ with carbon dioxide fixed at twice the PI concentration. We average the model output over the last $20 \mathrm{yr}$ of these simulations (years 201-220) and compare the climatological fields to their counterparts in that model's PI simulation. Theses runs will be referred to as the $2 \times \mathrm{CO}_{2}$ and PI runs, respectively.

\section{2) Results}

Averaged across all models, the doubling of $\mathrm{CO}_{2}$ results in a reduction in the hemispheric average planetary albedo of $-0.007 \pm 0.009$ in the $\mathrm{NH}$ and $-0.004 \pm 0.009$ in the $\mathrm{SH}$; the range quoted is 2 standard deviations. The intermodel spread in the change of hemispheric average planetary albedo is strongly correlated with the change in $\alpha_{P, \text { ATMOS }}$ in both hemispheres (Fig. 8a) with an $R^{2}$ value of 0.91 in the $\mathrm{NH}$ and 0.96 in the SH (both significant at the $99 \%$ confidence interval). In contrast, the hemispheric average $\alpha_{P, \mathrm{SURF}}$ change is not significantly correlated with the change in planetary albedo in either hemisphere (Fig. 8b). This is primarily because the intermodel spread in hemispheric average $\alpha_{P, \mathrm{ATMOS}}$ change is approximately 3 times as large of that in $\alpha_{P, \mathrm{SURF}}$. Although the change in hemispheric average $\alpha_{P, \mathrm{SURF}}$ is negative in all models (abscissa of Fig. 8b) because of decreasing ice and snow cover, the intermodel spread in the $\alpha_{P, \text { ATMOS }}$ overwhelms the change in surface contribution resulting in a total planetary albedo feedback that is ambiguous in sign among the ensemble members. This result is in qualitative agreement with Kato et al.'s 

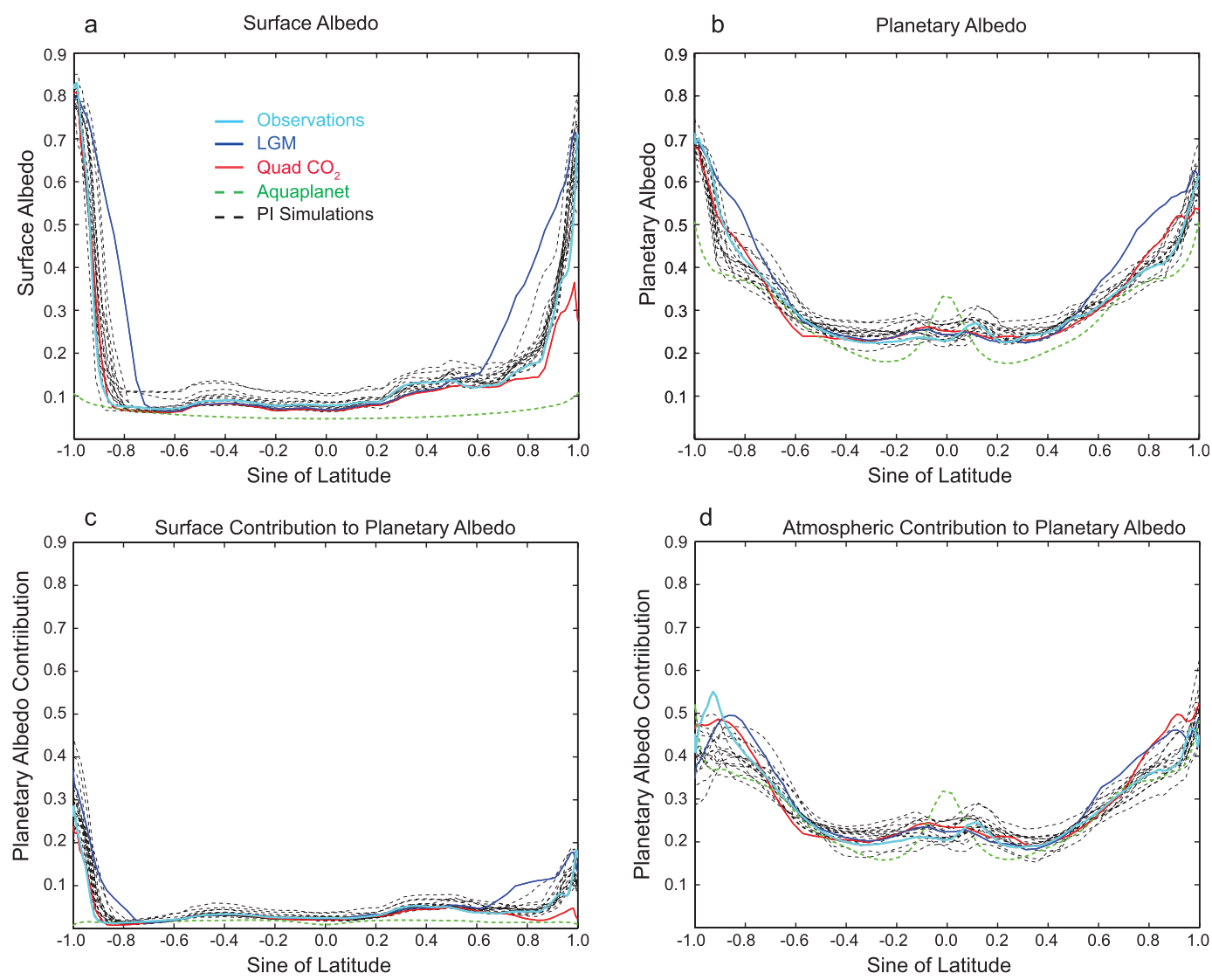

FIG. 6. Zonal annual mean (a) $\alpha$, (b) $\alpha_{P}$, (c) $\alpha_{P, \mathrm{SURF}}$, and (d) $\alpha_{P, \mathrm{ATMOS}}$ in the PI simulations from the CMIP3 models (dashed black lines). Also shown are the observations (solid light blue line) and model simulations of altered climate states (other colored lines).

(2006) conclusion that the recent decline in Arctic sea ice has a negligible signature on the Arctic radiative budget at the TOA because of atmospheric attenuation and uncertainty in cloud feedbacks.

The change in hemispheric average $\alpha_{P, \mathrm{SURF}}$ in the $2 \times \mathrm{CO}_{2}$ runs is well correlated with the change is hemispheric average surface albedo (Fig. 9a, $R^{2}=0.77$ in the $\mathrm{NH}$ and 0.87 in the $\mathrm{SH})$. This result can be understood by differentiating Eq. (5) with respect to $\alpha, R$, and $A$ :

$$
\begin{aligned}
\Delta\left(\alpha_{P, \mathrm{SURF}}\right)= & \frac{(1-R-A)^{2}}{(1-R \alpha)}\left(1+\frac{R \alpha}{1-R \alpha}\right) \Delta \alpha \\
& -\frac{2 \alpha(1-R-A)}{1-R \alpha} \Delta A-\frac{\alpha(1-R-A)}{1-R \alpha} \\
& \times\left(2-\alpha \frac{1-R-A}{1-R \alpha}\right) \Delta R .
\end{aligned}
$$

Equation (8) highlights that changes in $\alpha_{P, \mathrm{SURF}}$ are functions of both surface and atmospheric state; even if surface albedo remains constant in an altered climate state, the surface albedo contribution to planetary albedo could change by virtue of an atmospheric opacity change. Nonetheless, the changes in $\alpha_{P, \mathrm{SURF}}$ in the $2 \times \mathrm{CO} 2$ runs are dominated by the first term on the rhs of Eq. (8) because the fractional changes in $\alpha$ are much larger than the fractional changes in either $R$ or $A$ (not shown). Furthermore, because both $R$ and $\alpha$ are less than unity, $R \alpha /(1-R \alpha) \ll 1$, Eq. (8) can be approximated by

$$
\Delta \alpha_{P, \mathrm{SURF}} \approx \frac{(1-R-A)^{2}}{1-R \alpha} \Delta \alpha \equiv(1-\chi) \Delta \alpha .
$$

Equation (9) neglects how changes in atmospheric opacity and changes in multiple reflections impact $\alpha_{P, \mathrm{SURF}}$. It predicts that the changes in planetary albedo due to surface reflections are equal to the change in surface albedo modified by the atmospheric attenuation of surface albedo in the mean climate. If all climate models had the same atmospheric attenuation of surface albedo [and Eq. (9) held perfectly], then we would expect the change in $\alpha_{P, \mathrm{SURF}}$ in the $2 \times \mathrm{CO}_{2}$ to be proportional to $\Delta \alpha$ with a slope equal to $(1-\chi)$. The strong 


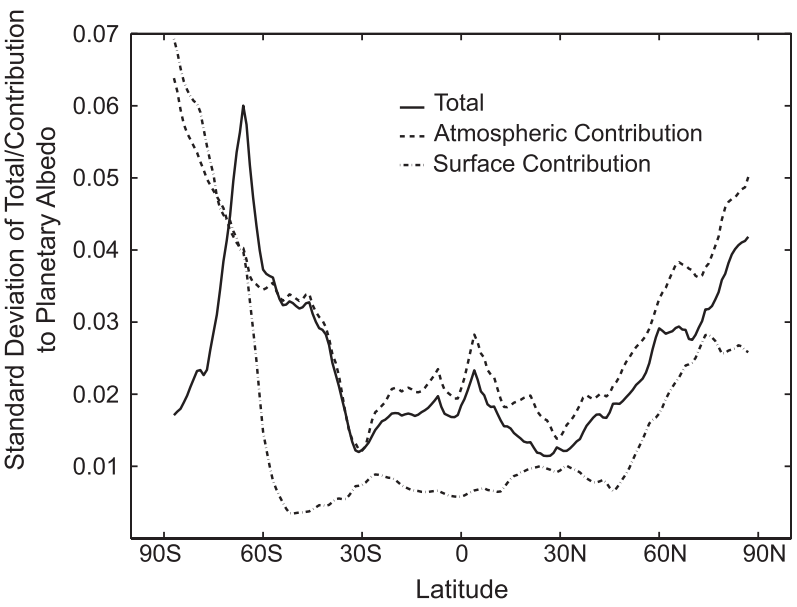

FIG. 7. The intermodel standard deviation of zonal average $\alpha_{P}$ (solid line), $\alpha_{P, \text { ATMOS }}$ (dashed line), and $\alpha_{P, \text { SURF }}$ (dashed-dotted line) for the CMIP3 PI simulations.

correlation in Fig. 9a indicates that the models have a similar atmospheric attenuation of surface albedo and that intermodel spread in the surface albedo feedback is primarily driven by model differences in the surface albedo response to warming. This result is in qualitative agreement with $\mathrm{Qu}$ and Hall's (2005) conclusion that the surface albedo feedback spread in climate models is due to spread in the simulated surface albedo changes and not in the intermodel spread of how surface albedo changes contribute to $F \uparrow$ тон. The slope of the best-fit lines in Fig. 9a are 0.34 in the $\mathrm{NH}$ and 0.30 in the $\mathrm{SH}$, suggesting that the change in planetary albedo associated with a change in surface albedo is approximately one-third of the magnitude of the change in surface albedo. This result is at odds with Qu and Hall's (2005) finding that the change in planetary albedo per unit change in surface albedo is approximately one-half. We have replicated Qu and Hall's result using their method on the datasets considered in this study suggesting the different quantitative conclusions are a consequence of methodological differences. The sensitivity of the atmospheric attenuation of surface albedo to the assumptions made in our simplified radiative transfer model (Fig. 1) is further discussed in appendix B.

Figure $9 \mathrm{~b}$ shows that there is no correlation between the change in planetary albedo and the change in surface albedo, averaged over a hemisphere. This is a consequence of the atmospheric opacity attenuating the contribution of surface albedo changes to $F \uparrow_{\text {TOA }}$ and the large intermodel spread in changes in atmospheric (cloud) reflectivity. This result suggests that changes in surface albedo associated with poleward retreat of the cryosphere relative to its location in the present day climate are a poor predictor of changes in planetary albedo because the shortwave cloud feedback uncertainties exert a more profound influence on the $F \uparrow_{\text {TOA }}$ than the direct radiative forcing of the surface albedo. These results agree with Trenberth and Fasullo (2009) who found that decreases in planetary albedo in the CMIP3 ensemble simulations for the A1B and $\mathrm{A} 2 \mathrm{CO}_{2}$ emission scenarios are primarily a consequence of reduced cloud cover.

\section{b. Simulations with specified surface albedo distributions}

In this section, we explore the relationship between surface albedo and planetary albedo in models of radically different climates that feature severely altered spatial patterns of surface albedo.

\section{1) MOdel RunS USED}

We consider three greatly different climate states: the Last Glacial Maximum (LGM), the equilibrated climate
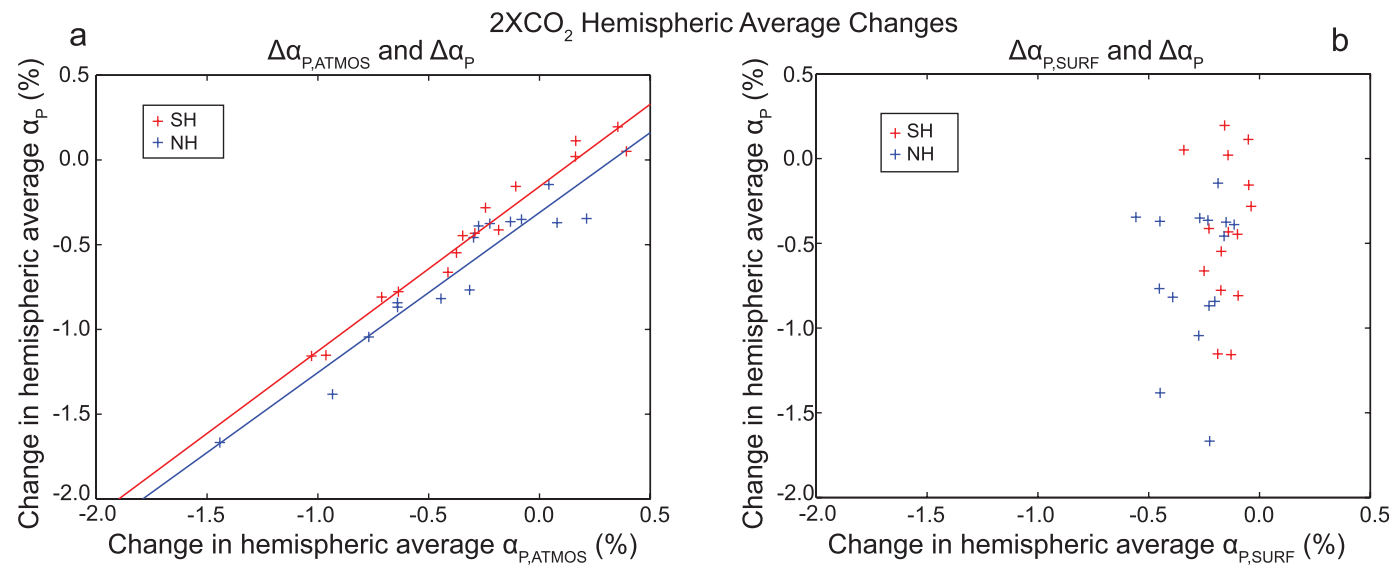

FIG. 8. (a) Change in hemispheric average planetary albedo in the $2 \times \mathrm{CO}_{2}$ runs (relative to the PI simulations) vs change in hemispheric average $\alpha_{P, \text { ATMOS}}$. The lines are the linear best fits in each hemisphere. (b) As in (a), but vs hemispheric average $\alpha_{P, \text { SURF }}$. All quantities are expressed as a percentage, where $1 \%$ corresponds to 0.01 units of albedo. 

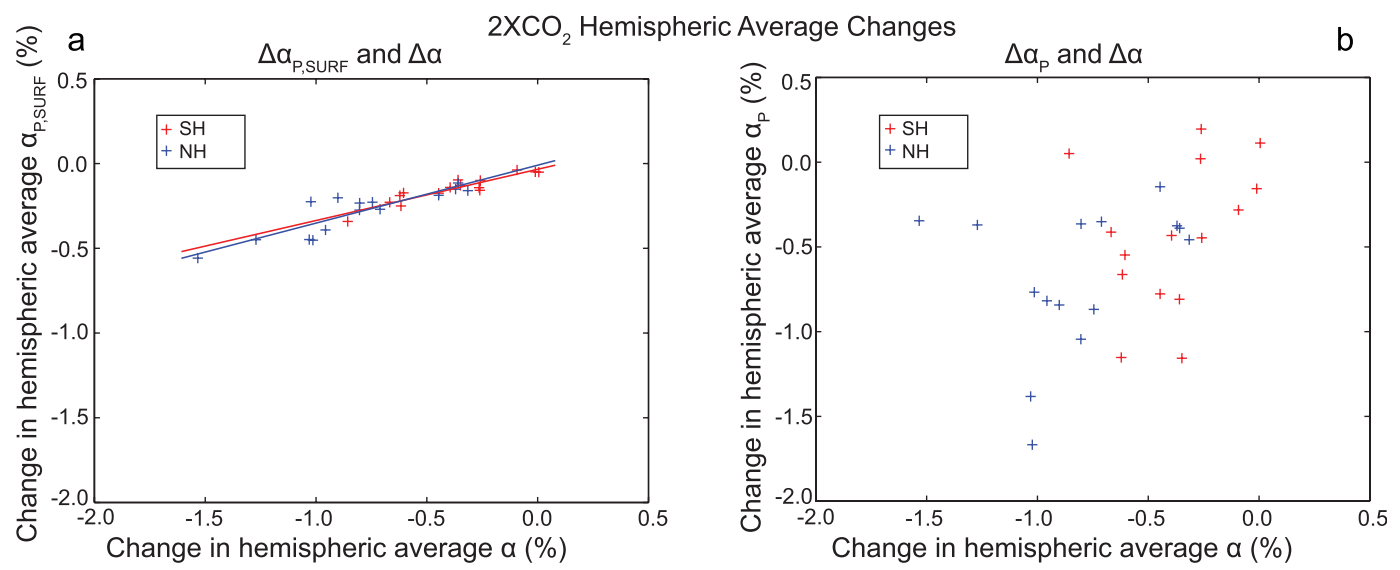

FIG. 9. (a) Change in hemispheric average $\alpha_{P, \text { SURF }}$ in the $2 \times \mathrm{CO}_{2}$ runs (relative to the PI simulations) vs change in hemispheric average $\alpha$. The lines are the linear best fits in each hemisphere. (b) Change in hemispheric average $\alpha_{P}$ in the $2 \times \mathrm{CO}_{2}$ runs (relative to the PI simulations) vs change in hemispheric average $\alpha$.

under 4 times the $\mathrm{PICO}_{2}$ concentration (QUAD), and an aquaplanet (AQUA) with no sea ice. The LGM simulation is performed with the National Center for Atmospheric Research's (NCAR) Community Atmosphere Model version 3 (CAM3) at a resolution of T42; it is forced with glacial orbital parameters and greenhouse gas concentrations, specified land ice albedo and topography and with sea ice concentrations and sea surface temperatures prescribed from the output of a fully coupled simulation of the LGM [see Li and Battisti (2008) for a description]. The QUAD simulation is also performed with CAM3 at a resolution of T42 with the greenhouse gas concentrations fixed at $1000 \mathrm{ppm}$ and with sea surface temperatures, sea ice concentrations, and snow extent prescribed as the output of an equilibrated coupled simulation. The LGM and QUAD runs are compared to a T42 CAM3 PI industrial run (the asterisks in Figs. 4 and 5) as opposed to the T63 coupled CCSM3 PI run that is included in the CMIP3 ensemble. The AQUA simulation is performed with Geophysical Fluid Dynamics Laboratory Atmosphere Model version 2 (GFDL AM2) coupled to a 50-m-depth slab ocean and sea ice formation is prohibited even if the temperature drops below the freezing point [this run is identical to Kang et al. (2008), except with a 50-m slab ocean]. All simulations are run for $50 \mathrm{yr}$ and we average the output over the last $20 \mathrm{yr}$ of a single ensemble member of each simulation. The AQUA simulation is compared to the fully coupled PI simulation included in the CMIP3 multimodel ensemble.

The prescribed spatial distribution of surface albedo varies widely in these runs (Figs. 6a, 10). The AQUA climate has almost no meridional gradient in surface albedo. (The small gradient in surface albedo is due to the angle of incidence dependence of ocean albedo.) Relative to the control PI climate simulated by the same model, the QUAD climate has a reduced high-latitude surface albedo because of a poleward shift in the ice edge, while the LGM run has greatly enhanced surface albedo in the $\mathrm{NH}$ midlatitudes mainly because of the presence of large continental ice sheets.

\section{2) Results}

While the changes in sea ice extent and in the distribution of land ice in the altered climate states greatly affect the surface albedo, the changes in planetary albedo are much smaller than the changes in surface albedo (Figs. 6, 10). For example, in the LGM simulations the surface albedo in the midlatitude $\mathrm{NH}$ increased by 0.25 (from $\sim 0.15$ in the PI simulation to $\sim 0.40$ in the LGM experiment), but the planetary albedo only increased by approximately 0.10 relative to the PI simulations because of a 0.06 increase in $\alpha_{P, \mathrm{SURF}}$ and a 0.04 increase in $\alpha_{P, \text { ATMOS }}$ (Fig. 10; the latter is due to more persistent clouds over the continental ice sheets).

In the global average, the planetary albedo increases from 0.300 in the PI to 0.320 in the LGM and is partitioned into a 0.012 increase in $\alpha_{P, \mathrm{SURF}}$ and a 0.008 increase in $\alpha_{P, \text { ATMOS }}$ (Table 4 ). The global average surface albedo increase of 0.040 exceeds the $\alpha_{P, \mathrm{SURF}}$ change by more than a factor of 3 , again suggesting that the impact of surface albedo changes on the TOA are attenuated by a factor of approximately 3 by atmospheric opacity. These results are in close agreement with T07, who found that the surface albedo changes over the ice sheets alone accounted for a 0.008 and 0.007 change in global average planetary albedo in two different simulations of the LGM. Our global average $\alpha_{P, \mathrm{SURF}}$ changes also include changes in surface albedo away from the ice sheets associated with a reduction in global sea level; we estimate 
GFDL Simulations
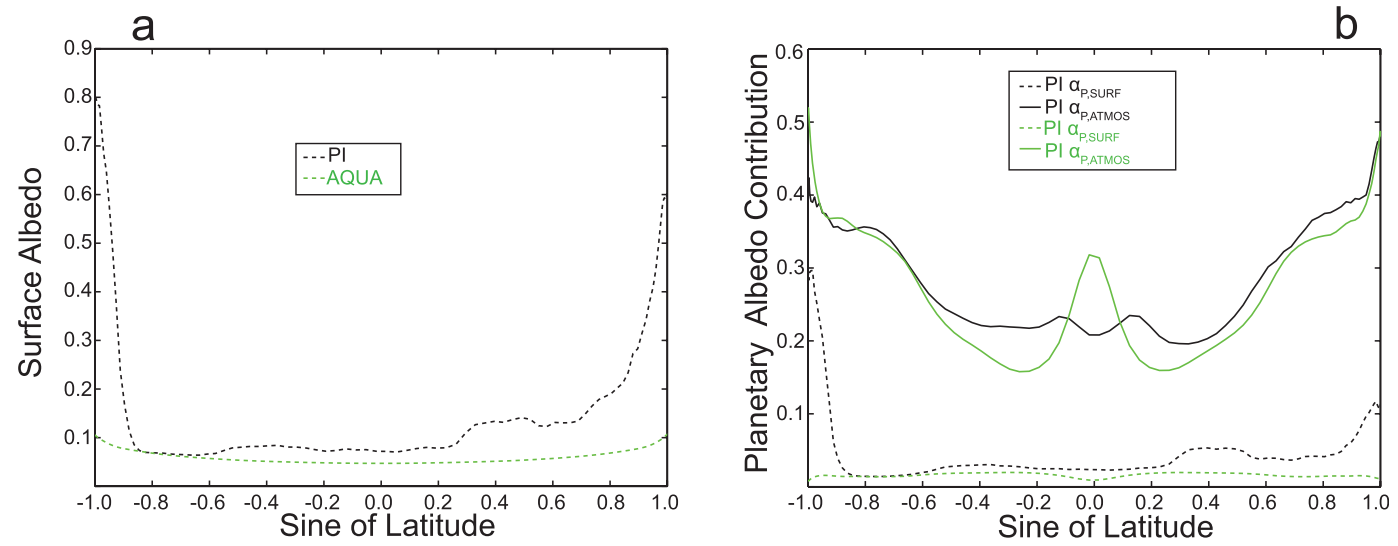

NCAR Simulations
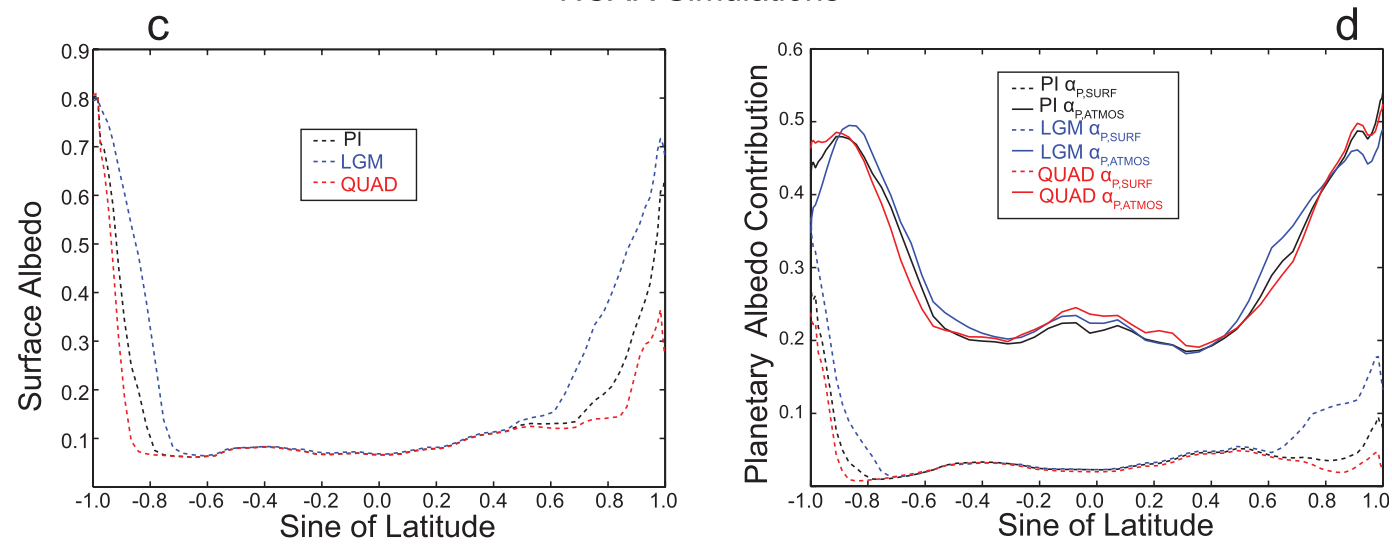

FIG. 10. (a) Zonal average $\alpha$ in the GFDL simulations of the PI (black) and AQUA (green). (b) Zonal average $\alpha_{P, \mathrm{ATMOS}}$, and $\alpha_{P, \mathrm{SURF}}$ in the GFDL simulations of the PI (black) and AQUA (green). (c),(d) As in (a) and (b), but for the NCAR simulations of the PI (black), QUAD (red), and LGM (blue).

this effect causes our global average $\alpha_{P, \text { SURF }}$ changes to be approximately $20 \%$ greater than that reported by $\mathrm{T} 07$.

In the AQUA simulation, the midlatitude and highlatitude total planetary albedo and $\alpha_{P, \mathrm{SURF}}$ decreases (relative to the PI simulations) by approximately one-third of the surface albedo change in the same regions. In the global average, however, the decrease in planetary albedo (relative to the GFDL PI simulation) is due to nearly equal decreases in $\alpha_{P, \mathrm{SURF}}$ and $\alpha_{P, \mathrm{ATMOS}}$ (Figs. 4a,b and Table 4). Further inspection (Fig. 10b) shows that the decrease in hemispheric average $\alpha_{P, \text { ATMOS }}$ in the AQUA simulation is almost entirely due to reduced atmospheric reflection in the subtropics. This surprising result is due to differences in the seasonal migration of the ITCZ between the AQUA and PI runs; the ITCZ remains on the equator during all seasons in the AQUA simulation, while the ITCZ migrates into the summer hemisphere in the PI simulation. This causes the subtropics to be cloudier during the sunny season in each hemisphere in the PI climate resulting in a larger annual average planetary albedo compared to the AQUA simulation in which the subtropics are relatively cloud free during all seasons.

It is worth discussing how the partitioning of planetary albedo in the altered climate state experiments

TABLE 4. Change in hemispheric average planetary albedo and the partitioning of the changes into atmospheric $\left(\alpha_{P, \mathrm{ATMOS}}\right)$ and surface $\left(\alpha_{P, \mathrm{SURF}}\right)$ contributions in the altered climate state simulations. Each difference is taken relative to the PI simulation in the same model used to simulate the altered climate state (and at the same resolution). The last two rows show the CMIP3 intermodel spread $(2 \sigma)$ of the same variables.

\begin{tabular}{ccccc}
\hline & & \multicolumn{3}{c}{ Hemispheric average $\alpha_{P}(\%)$} \\
\cline { 3 - 5 } Model & Hemisphere & $\alpha_{P}$ & $\alpha_{P, \text { ATMOS }}$ & $\alpha_{P, \text { SURF }}$ \\
\hline QUAD-PI & $\mathrm{NH}$ & -0.2 & +0.4 & -0.6 \\
& $\mathrm{SH}$ & -0.3 & +0.1 & -0.4 \\
LGM-PI & $\mathrm{NH}$ & +2.2 & +0.6 & +1.6 \\
& $\mathrm{SH}$ & +1.9 & +1.1 & +0.8 \\
AQUA-PI & $\mathrm{NH}$ & -4.2 & -1.8 & -2.4 \\
& $\mathrm{SH}$ & -3 & -1.5 & -1.5 \\
CMIP $2 \sigma$ & $\mathrm{NH}$ & 1.8 & 2.7 & 1.4 \\
& $\mathrm{SH}$ & 1.8 & 2.1 & 1.1 \\
\hline
\end{tabular}


would change if our partitioning methodology was systematically underestimating $\alpha_{P, \text { SURF }}$ relative to the "true" surface contribution to planetary albedo; in this case, $\alpha_{P, \text { ATMOS }}$ would be biased high relative to its "true" value [by Eq. (6)] especially over the cryospheric regions. If our $\alpha_{P, \mathrm{SURF}}$ values were biased low, we would expect to see large changes in $\alpha_{P, \text { ATMOS }}$ collocated with the regions of large changes in surface albedo (and with the same sign as the surface albedo change). This does not occur. In the AQUA simulation, the changes in $\alpha_{P, \mathrm{ATMOS}}$ are very small (except in the subtropics where the changes in surface albedo are also small, Fig. 10). In the QUAD simulation the changes in $\alpha_{P, \text { ATMOS }}$ and surface albedo have opposite signs in the cryosphere region; $\alpha_{P, \mathrm{ATMO}}$ increases in the high latitudes where the surface albedo has decreased relative to the PI and LGM simulations with the same model (Fig. 10). Thus, the partitioning of planetary albedo in the altered climate states suggests that the $\alpha_{P, \text { SURF }}$ values calculated by our methodology are not biased low.

\section{Summary and discussion}

We have partitioned the earth's planetary albedo into a component due to the reflection of incoming radiation by objects in the atmosphere $\alpha_{P, \text { ATMOS }}$ and a component due to reflection at the surface $\alpha_{P \text {,SURF. }}$ In the global average, the vast majority $(88 \%)$ of the observed planetary albedo is due to $\alpha_{P, \text { ATMOs. }}$. We demonstrate that the primary conclusions reached in this manuscript are robust to uncertainties in the observational datasets (appendix A) and the assumptions made in our simplified radiative transfer model (appendix B). The CMIP3 $\mathrm{PI}$ ensemble intermodel average planetary albedo is also primarily due to $\alpha_{P, \text { ATMOS }}(87 \%)$. The intermodel spread in global average planetary albedo is large, corresponding to radiative differences at the top of the atmosphere $(2 \sigma=$ $5.5 \mathrm{~W} \mathrm{~m}^{-2}$ ) that exceed the radiative forcing of doubling carbon dioxide. Intermodel differences in $\alpha_{P, \mathrm{ATMOS}}$ explain the majority $(>60 \%)$ of the intermodel spread in hemispheric average planetary albedo.

Surface albedo makes a small contribution to the global average planetary albedo because atmospheric processes attenuate the contribution of surface albedo to planetary albedo by a factor of approximately 3 . The atmosphere attenuates $\alpha_{P, \text { SURF }}$ relative to the surface albedo because the atmosphere is somewhat opaque to solar radiation and thus attenuates the amount of shortwave radiation incident on the TOA that reaches the surface and the amount of shortwave radiation reflected at the surface that is transmitted back to the TOA. As a consequence, the CMIP3 PI ensemble intermodel spread in planetary albedo is poorly correlated with the spread in surface albedo despite considerable intermodel differences in surface albedo.

Surface albedo decreases with global warming $\left(2 \times \mathrm{CO}_{2}\right.$ runs) in all models but the magnitude of the change varies significantly. However, the intermodel spread in the change in surface albedo is poorly correlated with the change in planetary albedo. The planetary albedo changes associated with global warming were found to be primarily due to changes in cloud albedo (93\% of the intermodel spread). This result is unsurprising given that cloud albedo plays the dominant role in setting the planetary albedo in the unperturbed climate and that the surface albedo's impact on the TOA radiative budget is strongly attenuated (approximately threefold) by the atmosphere.

Experiments with prescribed and severely altered surface albedo distributions associated with the Last Glacial Maximum and an aquaplanet demonstrated that changes in $\alpha_{P, \mathrm{SURF}}$ were approximately one-third the magnitude of the surface albedo changes because of atmospheric attenuation. Furthermore, the changes in planetary albedo in these experiments were due to nearly equal magnitude contributions from changes in cloud properties and the prescribed changes in surface albedo. This suggests that cloud feedbacks in greatly altered climate states are likely to play as important of a role in setting planetary albedo as even zeroth-order changes in surface albedo (Eastman and Warren 2010).

The most surprising result from this study is that the differences in planetary albedo in climates that have severely altered surface albedo are relatively modest compared to the spread in planetary albedo in the PI simulations of the CMIP3 models. For example, the difference in hemispheric average planetary albedo between the simulations of the PI world and the LGM is less than the spread in the PI simulations using the CMIP3 models. Similarly, the CMIP3 ensemble average change in planetary albedo due to global warming in the $2 \times \mathrm{CO}_{2}$ simulations is less than $30 \%$ of the intermodel spread in simulations of the present day climate. These results follow because planetary albedo is largely determined by atmospheric processes (cloud reflection) and there are large intermodel differences in cloud distributions in simulations of the PI climate. In contrast, planetary albedo is largely insensitive to surface processes. Thus, changes in surface albedo make a small contribution to planetary albedo changes relative to the large uncertainties in cloud distribution changes.

Our analysis has focused on global and hemispheric average planetary albedo. We note that surface albedo, $\alpha_{P, \mathrm{SURF}}$, and $\alpha_{P, \mathrm{ATMOS}}$ changes associated with global warming and paleoclimate states have a spatially nonuniform structure, often peaking at the high latitudes. 
The meridional structure of planetary albedo is inextricably linked to the equator-to-pole temperature gradient and the total heat transport in the climate system (Stone 1978) and these issues are currently under further investigation.

Acknowledgments. We acknowledge the modeling groups, the Program for Climate Model Diagnosis and Intercomparison (PCMDI) and the WCRP's Working Group on Coupled Modelling (WGCM) for their roles in making available the WCRP CMIP3 multimodel dataset. Support of this dataset is provided by the Office of Science, U.S. Department of Energy. Dargan Frierson ran the seasonal aquaplanet simulation utilized in this work and provided feedback on the discussion in this text. Camille Li ran and provided the model output for the LGM and QUAD $\mathrm{CO}_{2}$ runs. Stephen Warren's advice on the observational datasets used and comments were greatly appreciated. Tra Dinh and Tom Ackerman provided a radiative transfer model that aided in verifying our methodology and were also instrumental aids in understanding the radiative components of this work. This work was inspired by conversations with Gerard Roe, Kyle Armour, Ed Wrigglesworth Blanchard IV, Brian Rose, John Marshall, Valerio Lucarini, Rowan Sutton, Jonathan Gregory, and Patrick Taylor and benefitted from the critiques of three anonymous reviewers.

\section{APPENDIX A}

\section{Uncertainties in Observational Estimates of $\alpha_{P, A T M O S}$ and $\alpha_{P, S U R F}$}

The satellite-derived TOA and surface shortwave fluxes used in this study are both uncertain. Here we analyze how the uncertainty in these fields propagates to uncertainties in $\alpha_{P, \mathrm{SURF}}, \alpha_{P, \mathrm{ATMOS}}$, and $\alpha_{P}$.

The climatological CERES TOA shortwave fluxes have a total uncertainty of order $6 \mathrm{~W} \mathrm{~m}^{-2}$ or about $2 \%$ of the incident radiation (Loeb et al. 2009); the error in the CERES surface shortwave fluxes is approximately $5 \%$ (Kratz et al. 2010). We propagate these errors to obtain error estimates in $\alpha_{P, \mathrm{ATMOS}}$ and $\alpha_{P, \mathrm{SURF}}$ using a Monte Carlo simulation. At each grid point, we add a random error to the observed flux; the error is assumed to be Gaussian with a standard deviation of $2 \%$ of the observed flux for the TOA fluxes and $5 \%$ of the observed flux for the surface fluxes. We then take 100 random realizations of the radiative fluxes and calculate $\alpha_{P, \text { ATMOS }}$ and $\alpha_{P, \text { SURF }}$ using the methodology outlined in section 2 .

The $\alpha_{P, \text { ATMOS }}$ and $\alpha_{P, \text { SURF }}$ maps averaged over the 100 realizations are statistically indistinguishable from the maps calculated using the original data, suggesting

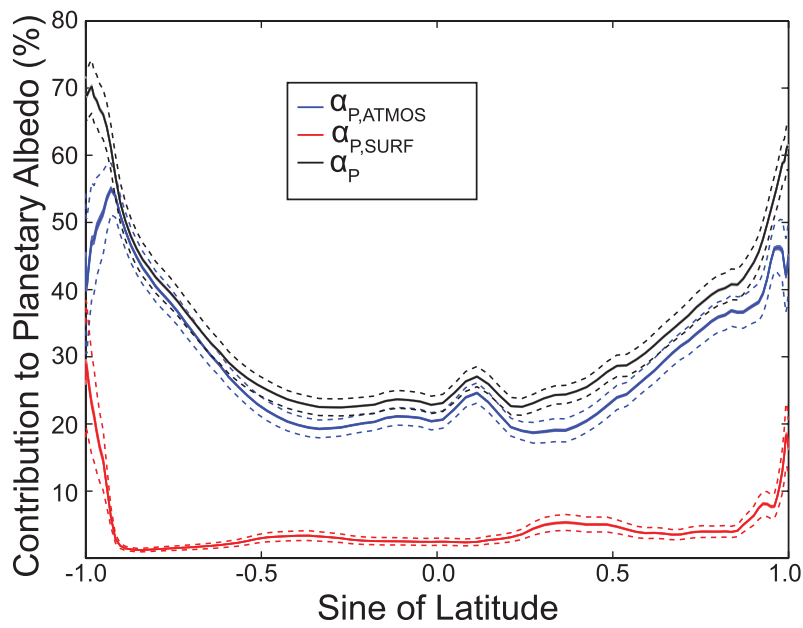

FIG. A1. Zonal average $\alpha_{P}, \alpha_{P, \text { ATMOS }}, \alpha_{P \text {,SURF }}$, and calculated from the observations and their uncertainties $(2 \sigma)$ estimated from the Monte Carlo simulations described in the text. The shaded areas are the uncertainty if the observational errors are random and uncorrelated at each grid point. The dashed lines are the uncertainty if the errors are systematic and perfectly correlated at all locations in each zonal band.

that our partitioning algorithm is sufficiently linear over the range of the uncertainty in the observed radiative fluxes. The standard deviation of the $\alpha_{P \text {,ATMOS }}$ over the 100 realizations is approximately $5 \%$ of the average $\alpha_{P, \text { ATMOS }}$ over most of the domain, while the standard deviation of $\alpha_{P, \mathrm{SURF}}$ is approximately $10 \%$ of the average $\alpha_{P, \mathrm{SURF}}$. The standard deviation of both $\alpha_{P, \mathrm{ATMOS}}$ and $\alpha_{P, \mathrm{SURF}}$ is significantly larger (reaching $25 \%$ of the average value) over the Greenland Ice Sheet and East Antarctica where the surface albedo is large and the higher-order reflections contribute to the partitioning algorithm (not shown).

We propagate the uncertainty in $\alpha_{P, \mathrm{ATMO}}$ and $\alpha_{P \text {,SURF }}$ onto the zonal averages (Fig. A1) with error bars representing two standard deviations. If we assume that the uncertainties are random, and uncorrelated at adjacent grid points, the uncertainties in the zonal average $\alpha_{P, \mathrm{ATMOS}}$ and $\alpha_{P, \mathrm{SURF}}$ (shaded areas) are less than 0.008 at all locations. In this case the standard deviation of the zonal average is approximately equal the standard deviation at a given grid point divided by the square root of the number of zonal grid points (zonal averaging reduces the uncertainty by more than a factor of 10). On the other hand, if the errors are systematic and have the same sign at all zonal locations, then the uncertainty in the zonal mean is equal to the zonal average uncertainty (dashed lines in Fig. A1 give $2 \sigma$ of this quantity). The true zonal average uncertainty is most likely somewhere in between these two estimates; observational errors at one grid point are expected to be correlated with those 
at their adjacent grid points with a spatial decorrelation scale that is regional (i.e., smaller than the zonal circumference assumed if the errors are systematic).

If the observational errors are systematic, the twostandard-deviation range of the global average $\alpha_{P, \text { ATMOS }}$ is $0.244-0.280$ (82\%-94\% of the total planetary albedo); the two-standard-deviation range of global average $\alpha_{P, \mathrm{SURF}}$ is $0.028-0.045(9 \%-15 \%$ of the total planetary albedo).

\section{APPENDIX B}

\section{Sensitivity of $\alpha_{P, \mathrm{ATM}}$ and $\alpha_{P, \text { SURF }}$ Partitioning Methodology}

The partitioning of planetary albedo into surface and atmospheric components presented in this paper is contingent on the assumptions underlying the simple single-layer shortwave radiative transfer model (Fig. 1 and section $2 b$ ). Specifically, we assume that 1) absorption and reflection of radiation is isotropic, 2) atmospheric absorption occurs during each pass through the atmosphere, and 3) absorption and reflection of shortwave radiation occurs at the same vertical level of the atmosphere. T07 partitioned the planetary albedo feedback between atmospheric and surface processes using a similar conceptual model except that they assumed that the absorption of shortwave radiation in the atmosphere occurred only during the first downward pass through the atmosphere (differs from our assumption 2) and that the absorption occurs above the level of atmospheric reflection (differs from our assumption 3; see their Fig. 1). Specifically, T07 assume that downwelling shortwave radiation in the atmospheric absorption bands is entirely depleted prior to arriving at the cloud-top level. (These assumptions allow the partitioning to be performed analytically.) In contrast, we assume that shortwave radiation reflected from the surface also experiences some absorption on its upward path toward the TOA because calculations using a radiative transfer model (Kato et al. 1999) show that the atmospheric absorption of solar radiation increases as the surface albedo is increased from 0 to 1 , indicating that solar radiation in the atmospheric absorption bands are not depleted in a single pass through the atmosphere. In this section, we explore the sensitivity of our results to these differing assumptions.

We apply the methodology of T07 to the observational and model datasets. We further illuminate the effects of the differing assumptions 2 and 3 by performing the planetary albedo partitioning with a model that assumes absorption occurs during the first pass through the atmosphere only, but that the absorption and reflection occur at the same level of the atmosphere. These three different models for partitioning planetary albedo are summarized in Table B1.

The partitioning of planetary albedo into $\alpha_{P, \mathrm{ATMOS}}$ and $\alpha_{P, \mathrm{SURF}}$ using each of these models is shown in Fig. B1 for both the observations and the ensemble average of the CMIP3 PI simulations. The model used in this study and the T07 model are in very close agreement in the partitioning of the observed planetary albedo at all latitudes. The T07 model finds lower values of $\alpha_{P, \mathrm{ATMOS}}$ (and consequentially, higher values of $\alpha_{P, \mathrm{SURF}}$ ) than the model used in this study at all latitudes. The average difference in $\alpha_{P, \text { ATMOS }}$ estimated using the two models is approximately $3 \%$ of the average $\alpha_{P, \mathrm{ATMO}}$ and does not exceed $10 \%$ at any latitude. Our model finds that $88 \%$ of the global average planetary albedo is due to $\alpha_{P, \mathrm{ATMOS}}$, whereas the T07 model finds a value of $85 \%$ (Table B1). The single absorption model finds smaller values of $\alpha_{P, \mathrm{ATMOS}}$ (and larger values of $\alpha_{P, \mathrm{SURF}}$ ) than the other two models; on average, the single absorption $\alpha_{P, \mathrm{ATMOS}}$ values are $4 \%$ smaller than the Taylor model and $9 \%$ smaller than the values found in this study.

The three methodologies yield similar results for the partitioning of planetary albedo between $\alpha_{P, \mathrm{ATMOS}}$ and $\alpha_{P, \text { SURF }}$ in the CMIP3 PI ensemble average. The smallest $\alpha_{P, \text { ATMOS }}$ values are obtained using the single absorption model and the model used in this study finds the highest $\alpha_{P, \mathrm{ATMOS}}$ (Fig. B1).

Although all three models give similar results for the partitioning of $\alpha_{P}$, the three models disagree on the magnitude of the atmospheric attenuation of surface albedo $K$. Our model finds that the atmosphere attenuates the surface albedo's contribution to the planetary albedo by $69 \%$ in the global average, whereas the Taylor and single absorption models find values of $61 \%$ and

TABLE. B1. Summary of the different simplified radiative transfer models used to calculate $\alpha_{P, \mathrm{ATMOS}}$ and $\alpha_{P, \mathrm{SURF}}$.

\begin{tabular}{lllcl}
\hline \hline \multicolumn{1}{c}{ Model name } & $\begin{array}{c}\text { Number of passes with } \\
\text { atmospheric absorption }\end{array}$ & \multicolumn{1}{c}{$\begin{array}{c}\text { Vertical location } \\
\text { of absorption }\end{array}$} & $\begin{array}{c}\text { Global average } \\
\alpha_{P, \text { ATMOS }}\end{array}$ & $\begin{array}{c}\text { Global average } \\
\alpha_{P, \text { SURF }}\end{array}$ \\
\hline This study & Infinite (all passes) & Collocated with reflection & $0.262(88 \%)$ & $0.036(12 \%)$ \\
T07 & 1 & Above reflection & $0.253(85 \%)$ & $0.045(15 \%)$ \\
Single absorption & 1 & Collocated with reflection & $0.246(83 \%)$ & $0.052(17 \%)$ \\
\hline
\end{tabular}



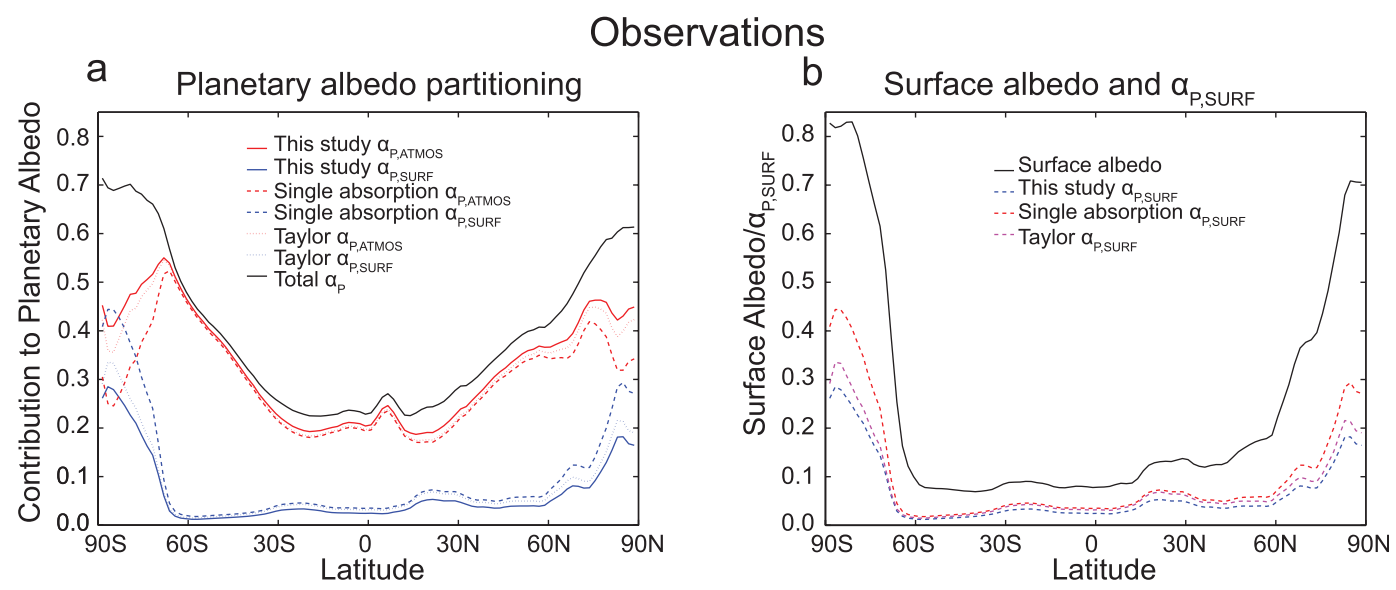

Inter-model Average
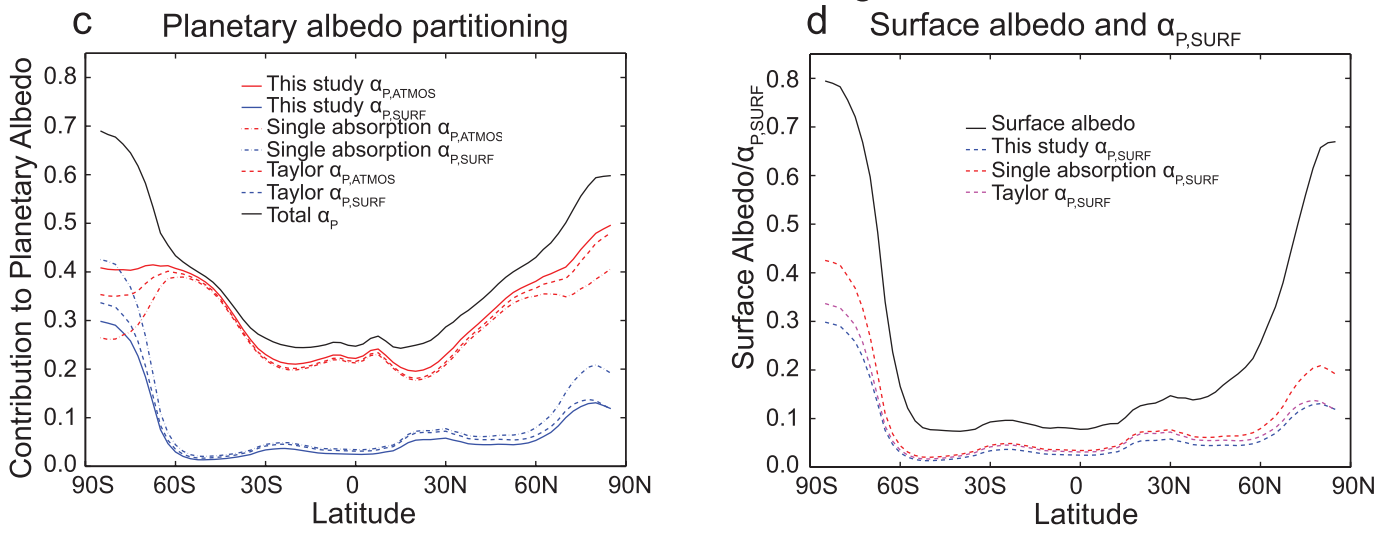

FIG. B1. (a) Planetary albedo partitioning in the observations using the model described in this study, the single absorption model, and the model of T07. (b) Surface albedo and surface contribution to planetary albedo using the three methods of planetary albedo partitioning. (c),(d) As in (a) and (b), but for the CMIP3 intermodel average.

$57 \%$. The differences in atmospheric attenuation between the three models are largest in the dry subtropics where atmospheric reflection is relatively small and thus absorption contributes to a larger fraction of the atmospheric opacity. Our model determines significantly larger values of attenuation in this region relative to the T07 and single absorption models because absorption increases the atmospheric opacity to upwelling shortwave radiation in our model and thus a smaller fraction of the radiation reflected by the surface escapes to space.

Although an evaluation of which model most accurately captures the radiative behavior of the atmosphere is beyond the scope of the present work, we will briefly comment on the source of differences in the results obtained using the three models, and why the T07 model yields results that are in closer agreement with our results than those using the single absorption model. The ratio of downwelling radiation at the surface and at the TOA is roughly equal to the atmospheric transmissivity (neglecting the effect of multiple reflections) and is constrained by the input radiative data used to partition the planetary albedo. In our model, the atmospheric transmissivity is $1-R-A$, whereas in Taylor's model ${ }^{1}$ it is $\left(1-R_{T}\right)\left(1-A_{T}\right)$, where the subscripts have been added to denote that the $R$ and $A$ values determined by the two models are not equivalent. Expanding out the opacity in T07's model, and knowing that values for $A$ and $A_{T}$ are similar (although $A$ is always greater than or equal to $A_{T}$ ), one can show that $R_{T}>R$. This is because the radiation reaching the cloud top has been depleted by absorption in the T07 model (and not in our model). Hence, clouds in the T07 model must reflect a larger fraction of the radiation incident at cloud top to satisfy the other radiative constraints imposed by the input data. The opacity to upwelling radiation in our model

\footnotetext{
${ }^{1}$ T07 call this quantity $\mu(1-\gamma)$, where $\mu$ is $\left(1-A_{T}\right)$ and $\gamma$ is reflection.
} 
is $1-R-A$, whereas in the T07 model it is $1-R_{T}$. The exclusion of absorption in atmospheric opacity to upwelling radiation in the $\mathrm{T} 07$ model is partially balanced by the fact that $R_{T}>R$. As a consequence, our model and the T07 model produce results that are in close agreement.

\section{REFERENCES}

Barkstrom, B., E. Harrison, G. Smith, R. Green, J. Kibler, and R. Cess, and The ERBE Science Team, 1989: Earth Radiation Budget (ERBE) archival and April 1985 results. Bull. Amer. Meteor. Soc., 70, 1254-1262.

Bender, F. A.-M., 2011: Planetary albedo in strongly forced climate, as simulated by the CMIP3 models. Theor. Appl. Climatol., doi:10.1007/s00704-011-0411-2, in press.

- - H. Rodhe, R. J. Charlson, A. M. L. Ekman, and N. Loeb, 2006: 22 views of the global albedo-Comparison between 20 GCMs and two satellites. Tellus, 58A, 320-330.

Bony, S., and Coauthors, 2006: How well do we understand climate change feedback processes? J. Climate, 19, 3445-3482.

Budyko, M. I., 1969: The effect of solar radiation variations on the climate of the Earth. Tellus, 21, 611-619.

Cahalan, R. F., W. Ridgway, W. J. Wiscombe, T. L. Bell, and J. B. Snider, 1994: The albedo of fractal stratocumulus clouds. J. Atmos. Sci., 51, 2434-2460.

Chou, M. D., and K. T. Lee, 1996: Parameterizations for the absorption of solar radiation by water vapor and ozone. J. Atmos. Sci., 53, 1203-1208.

Curry, J. A., W. B. Rossow, D. Randall, and J. L. Scramm, 1996: Overview of Arctic cloud radiation characteristics. J. Climate, 9, 1731-1762.

Eastman, R., and S. G. Warren, 2010: Interannual variations of Arctic cloud types in relation to sea ice. J. Climate, 23, 42164232.

Enderton, D., and J. Marshall, 2009: Controls on the total dynamical heat transport of the atmosphere and oceans. J. Atmos. Sci., 66, 1593-1611.

Fasullo, J. T., and K. E. Trenberth, 2008a: The annual cycle of the energy budget. Part I: Global mean and land-ocean exchanges. J. Climate, 21, 2297-2312.

- , and — 2008b: The annual cycle of the energy budget. Part II: Meridional structures and poleward transports. J. Climate, 21, 2313-2325.

Gorodetskaya, I. V., M. A. Cane, L.-B. Tremblay, and A. Kaplan, 2006: The effects of sea ice and land snow concentrations on planetary albedo from the Earth Radiation Budget Experiment. Atmos.-Ocean, 44, 195-205.

Hahn, C. J., and S. G. Warren, 2003: Cloud climatology for land stations worldwide, 1971-1996. Numerical data package NDP026D, Carbon Dioxide Information Analysis Center, 35 pp.

Hall, A., 2004: The role of surface albedo feedback in climate. J. Climate, 17, 1550-1568.

Hartmann, D. L., 1994: Global Physical Climatology. Academic Press, $411 \mathrm{pp}$.

Hummel, J. R., and R. A. Reck, 1979: A global surface albedo model. J. Appl. Meteor., 18, 239-253.

Kang, S. M., I. M. Held, D. M. W. Frierson, and M. Zhao, 2008: The response of the ITCZ to extratropical thermal forcing: Idealized slab-ocean experiments with a GCM. J. Climate, 21, 3521-3532.

Kato, S., T. P. Ackerman, J. H. Mather, and E. Clothiaux, 1999: The $k$-distribution method and correlated- $k$ approximation for a shortwave radiative transfer model. J. Quant. Spectrosc. Radiat. Transfer, 62, 109-121.

—, N. G. Loeb, P. Minnis, J. A. Francis, T. P. Charlock, D. A. Rutan, E. E. Clothiaux, and S. Sun-Mack, 2006: Seasonal and interannual variations of top-of-atmosphere irradiance and cloud cover over polar regions derived from CERES data set. Geophys. Res. Lett., 33, L19804, doi:10.1029/2006GL026685.

Kim, D., and V. Ramanathan, 2008: Solar radiation and radiative forcing due to aerosols. J. Geophys. Res., 113, D02203, doi:10.1029/2007JD008434.

Kratz, D. P., S. K. Gupta, A. C. Wilber, and V. E. Sothcott, 2010: Validation of the CERES edition 2B surface-only flux algorithms. J. Appl. Meteor. Climatol., 49, 164-180.

Li, C., and D. S. Battisti, 2008: Reduced Atlantic storminess during Last Glacial Maximum: Evidence from a coupled climate model. J. Climate, 21, 3561-3579.

Loeb, N. G., B. A. Wielicki, D. R. Doelling, G. L. Smith, D. F. Keyes, S. Kato, N. Manalo-Smith, and T. Wong, 2009: Towards optimal closure of the earth's top-of-atmosphere radiation budget. J. Climate, 22, 748-766.

Meehl, G. A., C. Covey, T. Delworth, M. Latif, B. McAvaney, J. F. B. Mitchell, R. J. Stouffer, and K. E. Taylor, 2007: The WCRP CMIP3 multi-model dataset: A new era in climate change research. Bull. Amer. Meteor. Soc., 88, 1383-1394.

Minnett, P. J., 1999: The influence of solar zenith angle and cloud type on cloud radiative forcing at the surface in the Arctic. J. Climate, 12, 147-158.

Moberg, A., D. M. Sonechkin, K. Holmgren, N. M. Datsenko, and W. Karlén, 2005: Highly variable Northern Hemisphere temperatures reconstructed from low- and high-resolution proxy data. Nature, 433, 613-617, doi:10.1038/nature03265.

Qu, X., and A. Hall, 2005: Surface contribution to planetary albedo variability in the cryosphere regions. J. Climate, 18, 5239-5252.

Roesch, A., 2006: Evaluation of surface albedo and snow cover in AR4 coupled climate models. J. Geophys. Res., 111, D15111, doi:10.1029/2005JD006473.

Rutan, D. A., F. G. Rose, N. M. Smith, T. P. Charlock, 2001: Validation data set for CERES surface and atmospheric radiation budget (SARB). WCRP/GEWEX Newsletter, Vol. 11, International GEWEX Project Office, Silver Spring, MD, 11-12.

Solomon, S., D. Qin, M. Manning, M. Marquis, K. Averyt, M. M. B. Tignor, H. L. Miller Jr., and Z. Chen, Eds., 2007: Climate Change 2007: The Physical Science Basis. Cambridge University Press, 996 pp.

Stone, P. H., 1978: Constraints on dynamical transports of energy on a spherical planet. Dyn. Atmos. Oceans, 2, 123-139.

Taylor, K. E., M. Crucifix, P. Braconnot, C. D. Hewitt, C. Doutriaux, A. J. Broccoli, J. F. B. Mitchell, and M. J. Webb, 2007: Estimating shortwave radiative forcing and response in climate models. J. Climate, 20, 2530-2543.

Trenberth, K. E., and J. T. Fasullo, 2009: Global warming due to increasing absorbed solar radiation. Geophys. Res. Lett., 36, L07706, doi:10.1029/2009GL037527.

,$- \ldots$, and J. Kiehl, 2009: Earth's global energy budget. Bull. Amer. Meteor. Soc., 90, 311-324.

Wielicki, B. A., R. D. Cess, M. D. King, D. A. Randall, and E. F. Harrison, 1995: Mission to planet Earth-Role of clouds and radiation in climate. Bull. Amer. Meteor. Soc., 76, 2125-2153.

— B. R. Barkstrom, E. F. Harrison, R. B. Lee, G. Louis Smith, and J. E. Cooper, 1996: Clouds and the Earth's Radiant Energy System (CERES): An Earth Observing System experiment. Bull. Amer. Meteor. Soc., 77, 853-868. 